\title{
The initial development of a jet caused by fluid, body and free surface interaction with a uniformly accelerated advancing or retreating plate. Part 2. Well-posedness and stability of the principal flow
}

\author{
M. T. Gallagher ${ }^{1}$, D. J. Needham ${ }^{1} \dagger$ and J. Billingham ${ }^{2}$ \\ ${ }^{1}$ School of Mathematics, University of Birmingham, Birmingham B15 2TT, UK \\ ${ }^{2}$ School of Mathematical Sciences, The University of Nottingham, University Park, \\ Nottingham NG7 2RD, UK
}

(Received 24 March 2016; revised 6 November 2017; accepted 22 December 2017; first published online 20 February 2018)

We consider the problem of a rigid plate, inclined at an angle $\alpha \in(0, \pi / 2)$ to the horizontal, accelerating uniformly from rest into, or away from, a semi-infinite strip of inviscid, incompressible fluid under gravity. Following on from Gallagher et al. (J. Fluid Mech., vol. 841, 2018, pp. 109-145) (henceforth referred to as GNB), it is of interest to analyse the well-posedness and stability of the principal flow with respect to perturbations in the initially horizontal free surface close to the plate contact point. In particular we find that the solution to the principal unperturbed problem, denoted by [IBVP] in GNB, is well-posed and stable with respect to perturbations in initial data in the region of interest, localised close to the contact point of the free surface and the plate, when the plate is accelerated with dimensionless acceleration $\sigma \geqslant-\cot \alpha$, while the solution to [IBVP] is ill-posed with respect to such perturbations in the initial data, when the plate is accelerated with dimensionless acceleration $\sigma<-\cot \alpha$. The physical source of the ill-posedness of the principal problem [IBVP], when $\sigma<-\cot \alpha$, is revealed to be due to the leading-order problem in the innermost region localised close to the initial contact point being in the form of a local Rayleigh-Taylor problem. As a consequence of this mechanistic interpretation we anticipate that, when the plate is accelerated with $\sigma<-\cot \alpha$, the inclusion of weak surface tension effects will restore well-posedness of the problem [IBVP] which will, however, remain temporally unstable.

Key words: instability, waves/free-surface flows

\section{Introduction}

In Gallagher, Needham \& Billingham (2018) (henceforth referred to as GNB) we considered the problem of the two-dimensional, irrotational flow generated by the 


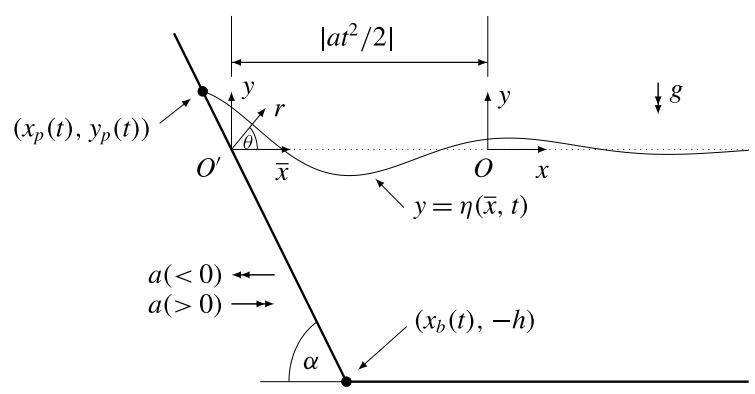

FIGURE 1. Definition sketch showing the displacement of the plate and free surface at time $t$.

uniform acceleration of a flat, rigid plate, inclined at an angle $\alpha \in(0, \pi / 2)$ to the horizontal, into or away from a uniform, horizontal strip of inviscid fluid under gravity. This work followed on from that of King \& Needham (1994), Needham, Billingham \& King (2007), Needham, Chamberlain \& Billingham (2008) and Needham (2012), a discussion of which can be found in GNB. In GNB we established that the structure of the solution to this principal free surface flow is characterised in terms of the parameters $\alpha$ and $\mu$ (where $\mu=1+\sigma \tan \alpha$, with $\sigma$ being the dimensionless acceleration of the plate), with a bifurcation in structure as $\mu$ changes sign. This change in local structure leads us to question the well-posedness and stability of the principal flow, and specifically, on short time scales $(\ll \sqrt{h / g})$, with respect to perturbations in the initially horizontal free surface close to the plate contact point and this question is examined in detail in this companion paper to GNB.

The structure of this paper is as follows. In $\S 2$ we formulate the mathematical problem through the introduction of initial perturbations in the horizontal free surface, close to the initial plate contact point. Subsequently $\S \S 3$ and 4 consider the well-posedness and stability of the principal flow (formulated as [IBVP] in GNB) determined by [IBVP] for plates inclined at all angles $\alpha \in(0, \pi / 2)$ with constant acceleration $\sigma$.

\section{Problem statement}

As discussed in the introduction, it is the purpose of this paper to address the well-posedness and stability of the principal flow described in detail in GNB. A definition sketch of the principal flow in GNB is given in figure 1. To this end we adopt all of the notation and terminology established in GNB without repetition (except where it is highlighted by $(\dagger)$ that a re-definition has been introduced, as, for example, after (3.31)). The principal flow was described in GNB by the initial boundary value problem denoted by [IBVP]. To analyse the well-posedness and stability of the problem [IBVP] with respect to initial perturbations in the horizontal free surface, close to the contact point, we introduce a perturbation on the zero initial data in [IBVP] in the following form, so that the initial conditions in [IBVP] are modified to

$$
\phi(\bar{x}, y, 0)=0, \quad(\bar{x}, y) \in \overline{\mathcal{D}}(0) ; \quad \eta(\bar{x}, 0)=\delta \eta_{0}\left(\frac{\bar{x}}{\delta^{m}}\right), \quad \bar{x} \geqslant 0,
$$


with $0<\delta \ll 1$ and $m>0$, whilst

$$
\eta_{0}(z)= \begin{cases}\bar{\eta}_{0}(z), & 0 \leqslant z \leqslant 1 \\ 0, & z>1,\end{cases}
$$

where $\bar{\eta}_{0}(z)$ is continuous with continuous derivative, and $\bar{\eta}_{0}(0)=\bar{\eta}_{0}(1)=\bar{\eta}_{0, z}(1)=0$. The form of the initial perturbation is chosen to localise the disturbance in an inner region close to the initial contact point, as it is the main purpose of this paper to examine the rapid localised interaction at the initial contact point between the surface perturbation and its principal development and, since the outer region will be passive in this evolution, the form (2.2) is used to eliminate the need for consideration of a perturbed form of the passive outer problem. The objective of interest is to study the evolution of the perturbation close to the initial contact point, within the innermost regions of the structure of the principal solution to [IBVP], as detailed in GNB. We will choose $m>0$ in (2.1) to achieve this end most effectively. Specifically, the principal flow problem [IBVP] studied in GNB will be referred to as well-posed when the solution to the perturbed problem approaches the solution to [IBVP] as $\delta \rightarrow 0$, uniformly on $\mathcal{S}_{\tau}=\cup_{\tau \in[0, T]}(\overline{\mathcal{D}}(\tau) \times\{\tau\})$ for each $T>0$ where $\tau$ is time scaled on a short time scale associated with $\delta$, as given in (3.2) or (4.3). In addition, when [IBVP] is well-posed, we refer to [IBVP] as stable when the solution to the perturbed problem approaches the solution to [IBVP] as $\tau \rightarrow \infty$, uniformly in $\overline{\mathcal{D}}(\tau)$; otherwise [IBVP] is said to be unstable. The structure of the solution to [IBVP], as detailed in GNB, determines that there are two cases to consider, which we address in turn. Specifically, as we shall see in what follows, we are interested in the initial dynamics of the flow and free surface close to the initial contact point, and we shall see that this flow develops rapidly on a time scale $t=O\left(\delta^{\alpha / \pi}\right)$ as $\delta \rightarrow 0$. Therefore the questions of stability and well-posedness must be addressed on the short time scale $t=O\left(\delta^{\alpha / \pi}\right)$, and, in particular, consider when local (to the initial contact point) well-posedness and stability properties emerge for $t \gg O\left(\delta^{\alpha / \pi}\right)$ (even though, in the bulk flow $t \ll 1)$. This becomes formally clear in the rescalings that follow.

\section{The case $(\alpha, \mu) \in\{(0, \pi / 2) \times \mathbb{R}\} \backslash\{(\pi / 4, \pi / 2) \times\{0\}\}$}

Following an examination of the inner asymptotic regions of [IBVP], as $t \rightarrow 0^{+}$, presented in GNB (see GNB (4.1), (4.3) and (4.7)), for the initial perturbation in the free surface to interact with the inner asymptotic region of the solution to [IBVP] as $t \rightarrow 0^{+}$, we require $t^{\pi / \alpha} \sim \delta$ and $t^{2} \sim \delta^{m}$, which, as $\delta \rightarrow 0$, determines

$$
t=O\left(\delta^{\alpha / \pi}\right) \quad \text { with } m=\frac{2 \alpha}{\pi} .
$$

In what follows, we will refer to the initially perturbed modification of [IBVP] as [IBVP]', and we will address [IBVP]' as $\delta \rightarrow 0$ with $t=O\left(\delta^{\alpha / \pi}\right)$. Thus we write

$$
t=\delta^{\alpha / \pi} \tau
$$

where $\tau=O(1)$ as $\delta \rightarrow 0$. We can now write [IBVP]' in terms of $\bar{x}, y, \phi, \eta, \tau$ and $\delta$, which becomes

$$
\begin{aligned}
\nabla^{2} \phi=0, & (\bar{x}, y) \in \mathcal{D}(\tau), \tau>0 \\
\nabla \phi \cdot \hat{\boldsymbol{n}}=\delta^{\alpha / \pi} \tau(\mu-1) \cos \alpha, & y=-\bar{x} \tan \alpha, \bar{x}_{p}(\tau)<\bar{x}<\cot \alpha, \tau>0
\end{aligned}
$$




$$
\begin{gathered}
\phi_{y}=0, \quad y=-1, \bar{x}>\cot \alpha, \tau>0 ; \\
\eta_{\tau}+\left[\delta^{\alpha / \pi} \phi_{\bar{x}}-\delta^{2 \alpha / \pi} \tau(\mu-1) \cot \alpha\right] \eta_{\bar{x}}-\delta^{\alpha / \pi} \phi_{y}=0, \\
y=\eta(\bar{x}, \tau), \quad \bar{x}>\bar{x}_{p}(\tau), \tau>0 ; \\
\phi_{\tau}-\delta^{2 \alpha / \pi} \tau(\mu-1) \cot \alpha \phi_{\bar{x}}+\delta^{\alpha / \pi} \frac{1}{2}|\nabla \phi|^{2}+\delta^{\alpha / \pi} \eta=0, \\
y=\eta(\bar{x}, \tau), \quad \bar{x}>\bar{x}_{p}(\tau), \tau>0 ; \\
\eta+\bar{x}_{p}(\tau) \tan \alpha=0, \quad \bar{x}=\bar{x}_{p}(\tau), \tau>0 ; \\
\eta \rightarrow 0, \quad \text { as } \bar{x} \rightarrow \infty, \tau>0 ;
\end{gathered}
$$

where $\eta_{0}: \mathbb{R} \rightarrow \mathbb{R}$ is given by (2.1) and $\hat{\boldsymbol{n}}$ is the unit vector normal to the plate pointing into the fluid region. Note that throughout $\dot{s}(t)=\sigma t$ and $\sigma$ has been eliminated in favour of $\mu$.

The structure of the solution to [IBVP] as $t \rightarrow 0^{+}$(as discussed in GNB) indicates that there will be two asymptotic regions in the solution to [IBVP]' as $\delta \rightarrow 0$ when $\tau=O(1)$. In the outer asymptotic region we have $(\bar{x}, y)(\in \overline{\mathcal{D}}(\tau))=O(1)$ and $\tau=O(1)$ as $\delta \rightarrow 0$, and we consider this first.

\subsection{Outer region for [IBVP]' when $\tau=O(1)$ as $\delta \rightarrow 0$}

We begin in an outer region in which $(\bar{x}, y)(\in \overline{\mathcal{D}}(\tau))=O(1)$ and $\tau=O(1)$ as $\delta \rightarrow 0$, after which we will require an inner region, in which $(\bar{x}, y)=o(1)$ and $\tau=O(1)$ as $\delta \rightarrow 0$, in order to capture the initial interaction between the plate and the perturbed fluid free surface. We first observe that the initial conditions (3.11) and (3.12) require that $\eta, \phi=o(1)$ as $\delta \rightarrow 0$ in the outer region. Specifically, conditions (3.4) and (3.6) require that $\phi=O\left(\delta^{\alpha / \pi}\right)$ and $\eta=O\left(\delta^{2 \alpha / \pi}\right)$ as $\delta \rightarrow 0$. Thus we introduce the asymptotic expansions

$$
\begin{gathered}
\phi(\bar{x}, y, \tau)=\delta^{\alpha / \pi}(\mu-1) \cos \alpha \phi^{\prime}(\bar{x}, y, \tau)+O\left(\delta^{2 \alpha / \pi}\right), \\
\eta(\bar{x}, \tau)=\delta^{2 \alpha / \pi} \eta^{\prime}(\bar{x}, \tau)+O\left(\delta^{3 \alpha / \pi}\right),
\end{gathered}
$$

as $\delta \rightarrow 0$ in the outer region, with the factor $(\mu-1) \cos \alpha$ in (3.13) included for algebraic convenience at a later stage. Here we note that the notation ' is not to be confused with the indication of dimensionality in GNB, nor does it refer to differentiation. Substituting the expansions (3.13) and (3.14) into [IBVP]', we obtain the leading-order evolution problem in the outer region for $\phi^{\prime}$, namely

$$
\begin{gathered}
\nabla^{2} \phi^{\prime}=0, \quad(\bar{x}, y) \in \mathcal{D}(0), \tau>0 ; \\
\nabla \phi^{\prime} \cdot \hat{\boldsymbol{n}}=\tau, \quad y=-\bar{x} \tan \alpha, 0<\bar{x}<\cot \alpha, \tau>0 ; \\
\phi_{y}^{\prime}=0, \quad y=-1, \bar{x}>\cot \alpha, \tau>0 ; \\
\phi_{\tau}^{\prime}=0, \quad y=0, \bar{x}>0, \tau>0 ; \\
\left|\nabla \phi^{\prime}\right| \rightarrow 0 \quad \begin{array}{l}
\text { as } \bar{x} \rightarrow \infty, \quad \text { uniformly for }-1 \leqslant y \leqslant 0, \tau>0 ; \\
\phi^{\prime}(\bar{x}, y, 0)=0, \quad(\bar{x}, y) \in \overline{\mathcal{D}}(0) ;
\end{array}
\end{gathered}
$$

with $\eta^{\prime}$ then given by

$$
\begin{gathered}
\eta_{\tau}^{\prime}(\bar{x}, \tau)=\frac{1}{2}(\mu-1) \cos \alpha \phi_{y}^{\prime}(\bar{x}, 0, \tau), \quad \bar{x} \geqslant 0, \tau>0 \\
\eta^{\prime}(\bar{x}, 0)=0, \quad \bar{x} \geqslant 0 .
\end{gathered}
$$


Note that the free surface perturbation is absent in (3.22) as the perturbation is localised in the inner region. We next introduce $\bar{\phi}^{\prime}(\bar{x}, y)$ and $\bar{\eta}^{\prime}(\bar{x})$ by writing

$$
\phi^{\prime}(\bar{x}, y, \tau)=\tau \bar{\phi}^{\prime}(\bar{x}, y), \quad \eta^{\prime}(\bar{x}, \tau)=\tau^{2} \bar{\eta}^{\prime}(\bar{x}) .
$$

The resulting problem for $\bar{\phi}^{\prime}$ and $\bar{\eta}^{\prime}$, obtained after substituting (3.23) into (3.15)(3.22), is the same as the outer region problem which was solved and discussed in GNB, and as such details are omitted here. We thus have that $\bar{\phi}^{\prime}$ is given, for some real constants $A_{n}, B_{n}$ and $C_{n}(n=0,1,2, \ldots)$, by

$$
\bar{\phi}^{\prime}(r, \theta)=\frac{r \sin \theta}{\cos \alpha}+\sum_{n=0}^{\infty} A_{n} r^{(n+1 / 2) \pi / \alpha} \sin ((n+1 / 2) \pi \theta / \alpha),
$$

for $0 \leqslant r<\operatorname{cosec} \alpha,-\alpha \leqslant \theta \leqslant 0$, where $\bar{x}=r \cos \theta$ and $y=r \sin \theta$, whilst

$$
\bar{\phi}^{\prime}(\rho, \bar{\theta})=\frac{\rho \cos \bar{\theta}}{\sin \alpha}+\sum_{n=0}^{\infty} B_{n} \rho^{n \pi /(\pi-\alpha)} \cos \left(\frac{n \pi \bar{\theta}}{\pi-\alpha}\right),
$$

for $0 \leqslant \rho<1,0 \leqslant \bar{\theta} \leqslant \pi-\alpha$, where $\bar{x}-\cot \alpha=\rho \cos \bar{\theta}$, and $y+1=\rho \sin \bar{\theta}$, and finally

$$
\bar{\phi}^{\prime}(\bar{x}, y)=\sum_{n=0}^{\infty} C_{n} \mathrm{e}^{-(n+1 / 2) \pi \bar{x}} \sin ((n+1 / 2) \pi y),
$$

for $\bar{x}>\cot \alpha,-1 \leqslant y \leqslant 0$. It then follows from (3.21), (3.24) and (3.26), that

$$
\bar{\eta}^{\prime}(\bar{x})=\frac{1}{2} \sigma \tan \alpha+\frac{1}{2} \sigma \sin \alpha \sum_{n=0}^{\infty}(n+1 / 2) \frac{\pi}{\alpha} A_{n} \bar{x}^{(n+1 / 2) \pi / \alpha-1},
$$

for $0 \leqslant \bar{x}<\operatorname{cosec} \alpha$, and

$$
\bar{\eta}^{\prime}(\bar{x})=\frac{1}{2} \sigma \sin \alpha \sum_{n=0}^{\infty}(n+1 / 2) \pi C_{n} \mathrm{e}^{-(n+1 / 2) \pi \bar{x}},
$$

for $\bar{x} \geqslant \operatorname{cosec} \alpha$. We observe from (3.24) and (3.27) that, as $(\bar{x}, y) \rightarrow(0,0)$,

$$
\begin{aligned}
\bar{\phi}^{\prime}(r, \theta)= & \frac{r \sin \theta}{\cos \alpha}+A_{0}(\alpha) r^{\pi / 2 \alpha} \sin \frac{\pi \theta}{2 \alpha}+O\left(r^{3 \pi / 2 \alpha}\right), \\
& \text { as } r \rightarrow 0^{+}, \text {with }-\alpha \leqslant \theta \leqslant 0, \\
\bar{\eta}^{\prime}(\bar{x})= & \frac{1}{2}(\mu-1)+A_{0}(\alpha) \frac{\pi}{4 \alpha}(\mu-1) \cos \alpha \bar{x}^{\pi / 2 \alpha-1}+O\left(\bar{x}^{3 \pi / 2 \alpha-1}\right), \\
& \text { as } \bar{x} \rightarrow 0^{+},
\end{aligned}
$$

where $r$ and $\theta$ are the polar coordinates defined above, and $A_{0}(\alpha)(<0)$ as given in GNB (see GNB (3.10)). Equation (3.30) reveals a weak singularity in $\bar{\eta}_{\bar{x}}^{\prime}(\bar{x})$ as $\bar{x} \rightarrow 0^{+}$. This singular behaviour as $\bar{x} \rightarrow 0^{+}$is compounded in higher-order terms in the outer expansions (3.13) and (3.14), and so the regularity conditions (see GNB (2.16)) fail to be satisfied by the outer region asymptotic expansions in a neighbourhood of the initial intersection point of the plate and the free surface, where $(\bar{x}, y)=o(1)$ as $\delta \rightarrow 0$. Therefore, in order to capture the full regularity in the neighbourhood of the intersection point of the plate and the free surface, the introduction of an inner region is required. 
3.2. Inner region for $[I B V P]^{\prime}$ when $\tau=O(1)$ as $\delta \rightarrow 0$

We have $(\bar{x}, y)=O(\nu(\delta))$, with $\nu(\delta)=o(1)$ as $\delta \rightarrow 0$ in the inner region. It then follows from (3.14), (3.23) and (3.30) that $\eta=O\left(\delta^{2 \alpha / \pi}\right)$ as $\delta \rightarrow 0$ in the inner region, so that, to capture the free surface in the inner region, we must take $v(\delta)=O\left(\delta^{2 \alpha / \pi}\right)$ as $\delta \rightarrow 0$; therefore, without loss of generality, we set $\nu(\delta)=\delta^{2 \alpha / \pi}$. An examination of (3.13), (3.23), and (3.29) then requires that $\phi=O\left(\delta^{2 \alpha / \pi}\right)$ as $\delta \rightarrow 0$ in the inner region. Thus we introduce scaled inner region coordinates $(X, Y)$ by

$$
\bar{x}=\delta^{2 \alpha / \pi} X, \quad y=\delta^{2 \alpha / \pi} Y,
$$

with $(X, Y)=O(1)$ as $\delta \rightarrow 0(\dagger)$. The plate is located in the inner region at $Y=-X \tan \alpha$, with the contact point denoted by $(X, Y)=\left(X_{p}(\tau), Y_{p}(\tau)\right)$, where $\bar{x}_{p}(\tau)=\delta^{2 \alpha / \pi} X_{p}(\tau)$ and $y_{p}(\tau)=\delta^{2 \alpha / \pi} Y_{p}(\tau)(\dagger)$. We now write the free surface and velocity potential in the inner region as

$$
\begin{gathered}
\eta(X, \tau)=\delta^{2 \alpha / \pi} \eta_{I}(X, \tau), \quad X \geqslant X_{p}(\tau), \tau \geqslant 0 \\
\phi(X, Y, \tau)=\delta^{3 \alpha / \pi} \phi_{I}(X, Y, \tau), \quad X \geqslant X_{p}(\tau),-X \tan \alpha \leqslant Y \leqslant \eta_{I}(X, \tau), \tau \geqslant 0 ;
\end{gathered}
$$

where $\eta_{I}(X, \tau), \phi_{I}(X, Y, \tau)=O(1)$ as $\delta \rightarrow 0(\dagger)$. The inner region expansions are introduced as

$$
\begin{gathered}
\eta_{I}(X, \tau)=\widetilde{\eta}_{0}^{\prime}(X, \tau)+\delta^{1-2 \alpha / \pi} \widetilde{\eta}_{1}^{\prime}(X, \tau)+o\left(\delta^{1-2 \alpha / \pi}\right), \\
\phi_{I}(X, Y, \tau)=\widetilde{\phi}_{0}^{\prime}(X, Y, \tau)+\delta^{1-2 \alpha / \pi} \widetilde{\phi}_{1}^{\prime}(X, Y, \tau)+o\left(\delta^{1-2 \alpha / \pi}\right),
\end{gathered}
$$

as $\delta \rightarrow 0$ with $(X, Y)=O(1)$, where the form of the correction terms have been deduced from (3.31), together with (3.14), (3.23) and (3.30). It follows from (3.31) and (3.34) that the free surface in the inner region is located at

$$
Y(X, \tau)=\widetilde{\eta}_{0}^{\prime}(X, \tau)+\delta^{1-2 \alpha / \pi} \widetilde{\eta}_{1}^{\prime}(X, \tau)+o\left(\delta^{1-2 \alpha / \pi}\right), \quad X>X_{p}(\tau), \tau \geqslant 0,
$$

Hence we must expand

$$
X_{p}(\tau)=X_{0}(\tau)+\delta^{1-2 \alpha / \pi} X_{1}(\tau)+o\left(\delta^{1-2 \alpha / \pi}\right), \quad \tau \geqslant 0,
$$

as $\delta \rightarrow 0$, after which $Y_{p}(\tau)=-X_{p}(\tau) \tan \alpha(\dagger)$.

We now write [IBVP]' in terms of the inner variables and substitute from (3.34) and (3.35). The problem obtained at leading order, when supplemented with matching conditions to the outer region (obtained through Van Dyke's matching principle (Van Dyke 1964)), has the solution

$$
\begin{gathered}
\widetilde{\phi}_{0}^{\prime}(X, Y, \tau)=\tau(\mu-1) Y+\frac{1}{6} \tau^{3}(1-\mu)(2 \mu-1), \\
X \geqslant X_{0}(\tau),-X \tan \alpha \leqslant Y \leqslant \frac{1}{2} \tau^{2}(\mu-1), \tau \geqslant 0 ; \\
\widetilde{\eta}_{0}^{\prime}(X, \tau)=\frac{1}{2} \tau^{2}(\mu-1), \quad X \geqslant X_{0}(\tau), \tau \geqslant 0 ; \\
X_{0}(\tau)=-\frac{1}{2} \tau^{2}(\mu-1) \cot \alpha, \quad \tau \geqslant 0 .
\end{gathered}
$$

It follows from (3.38) and (3.39), with (3.31)-(3.35), that the regularity conditions (see GNB (2.16)) are satisfied at leading order in the inner region. We now formulate the problem at $O\left(\delta^{1-2 \alpha / \pi}\right)$, where it is convenient to introduce the coordinates $(\bar{X}, \bar{Y})$ as

$$
X=-\frac{1}{2} \tau^{2}(\mu-1) \cot \alpha+\bar{X}, \quad Y=\frac{1}{2} \tau^{2}(\mu-1)+\bar{Y},
$$


which is simply a shift of origin from the original inner coordinates $(X, Y)$. We next write

$$
\widetilde{\eta}_{1}^{\prime}(\bar{X}, \tau)=\left.\widetilde{\eta}_{1}^{\prime}(\bar{X}, \tau)\right|_{0}+H^{\prime}(\bar{X}, \tau), \quad \widetilde{\phi}_{1}^{\prime}(\bar{X}, \bar{Y}, \tau)=\left.\widetilde{\phi}_{1}^{\prime}(\bar{X}, \bar{Y}, \tau)\right|_{0}+\Phi^{\prime}(\bar{X}, \bar{Y}, \tau), \quad(3.42 a, b)
$$

where $\left.\widetilde{\eta}_{1}^{\prime}\right|_{0}$ and $\left.\widetilde{\phi}_{1}^{\prime}\right|_{0}$ are given by

$$
\begin{gathered}
\left.\widetilde{\eta}_{1}^{\prime}(\bar{X}, \tau)\right|_{0}=A_{0}(\alpha)(\mu-1) \cos \alpha \tau^{\pi / \alpha-2} \widehat{\eta}\left(\frac{\bar{X}}{\tau^{2}}\right), \quad \bar{X} \geqslant 0, \tau>0, \\
\left.\widetilde{\phi}_{1}^{\prime}(\bar{X}, \bar{Y}, \tau)\right|_{0}=A_{0}(\alpha)(\mu-1) \cos \alpha \tau^{\pi / \alpha-2} \widehat{\phi}\left(\frac{\bar{X}}{\tau^{2}}, \frac{\bar{Y}}{\tau^{2}}\right), \\
\bar{X} \geqslant 0,-\bar{X} \tan \alpha \leqslant \bar{Y} \leqslant 0, \tau>0,
\end{gathered}
$$

and $\widehat{\eta}$ and $\widehat{\phi}$ are solutions to the boundary value problem in GNB (see (3.10)-(4.16)) when written in the similarity coordinates $(\bar{X}, \bar{Y}) \rightarrow \tau^{-2}(\bar{X}, \bar{Y})$. Rewriting the problem at $O\left(\delta^{1-2 \alpha / \pi}\right)$ in terms of $H^{\prime}$ and $\Phi^{\prime}$ we obtain

$$
\begin{gathered}
\bar{\nabla}^{2} \Phi^{\prime}=0, \quad \bar{X}>0,-\bar{X} \tan \alpha<\bar{Y}<0, \tau>0 ; \\
\bar{\nabla} \Phi^{\prime} \cdot \hat{\boldsymbol{n}}=0, \quad \bar{X}>0, \bar{Y}=-\bar{X} \tan \alpha, \tau>0 ; \\
H_{\tau}^{\prime}-\Phi_{\bar{Y}}^{\prime}=0, \quad \bar{X}>0, \bar{Y}=0, \tau>0 ; \\
\Phi_{\tau}^{\prime}+\mu H^{\prime}=0, \quad \bar{X}>0, \bar{Y}=0, \tau>0 ; \\
\Phi^{\prime}(\bar{R}, \theta, \tau) \rightarrow 0 \quad \text { as } \bar{R} \rightarrow \infty,-\alpha<\theta<0, \tau>0 ; \\
H^{\prime}(\bar{X}, \tau) \rightarrow 0 \quad \text { as } \bar{X} \rightarrow \infty, \tau>0 ; \\
\Phi^{\prime}(\bar{R}, \theta, 0)=0, \quad \bar{R} \geqslant 0,-\alpha \leqslant \theta \leqslant 0 ; \\
H^{\prime}(\bar{X}, 0)=\eta_{0}(\bar{X}), \quad \bar{X} \geqslant 0 .
\end{gathered}
$$

Here $\bar{\nabla}=(\partial / \partial \bar{X}, \partial / \partial \bar{Y})$ and we have, for convenience, introduced polar coordinates $(\bar{R}, \theta)$, given by $\bar{X}=\bar{R} \cos \theta, \bar{Y}=\bar{R} \sin \theta(\dagger)$. We begin our investigation of the problem (3.45)-(3.52) in the case $(\alpha, \mu) \in(0, \pi / 2) \times(\mathbb{R} \backslash\{0\})$. We seek solutions of the form

$$
\Phi^{\prime}(\bar{X}, \bar{Y}, \tau)=\mathrm{e}^{-\lambda \tau} \bar{\Phi}^{\prime}(\bar{X}, \bar{Y}), \quad H^{\prime}(\bar{X}, \tau)=\mathrm{e}^{-\lambda \tau} \bar{H}^{\prime}(\bar{X}),
$$

where $\lambda \in \mathbb{C}$, which leads to a decoupled linear harmonic boundary value problem for $\bar{\Phi}^{\prime}$, given by

$$
\begin{gathered}
\bar{\nabla}^{2} \bar{\Phi}^{\prime}=0, \quad \bar{X}>0,-\bar{X} \tan \alpha<\bar{Y}<0 ; \\
\bar{\nabla}^{\prime} \cdot \hat{\boldsymbol{n}}=0, \quad \bar{X}>0, \bar{Y}=-\bar{X} \tan \alpha ; \\
\bar{\Phi}_{\bar{Y}}^{\prime}-k \bar{\Phi}^{\prime}=0, \quad \bar{X}>0, \bar{Y}=0 ; \\
\bar{\Phi}^{\prime}, \bar{\nabla} \bar{\Phi}^{\prime} \quad \text { bounded as } \bar{R} \rightarrow \infty, \text { uniformly for }-\alpha<\theta<0 ;
\end{gathered}
$$

where $k=-\lambda^{2} / \mu$ (for $\mu \neq 0$ ). Although unnecessary, if required we obtain $\bar{H}^{\prime}$ from

$$
\bar{H}^{\prime}(\bar{X})=\frac{\lambda}{\mu} \bar{\Phi}^{\prime}(\bar{X}, 0), \quad \bar{X} \geqslant 0 .
$$


Here, according to the regularity conditions (see GNB (2.16)), we require that solutions to (3.54)-(3.57), $\bar{\Phi}^{\prime}: \overline{\mathcal{G}}_{\infty} \rightarrow \mathbb{C}$, have regularity

$$
\bar{\Phi}^{\prime} \in C^{1}\left(\overline{\mathcal{G}}_{\infty}\right) \cap C^{2}\left(\mathcal{G}_{\infty}\right),
$$

where

$$
\mathcal{G}_{\infty}=\{(\bar{X}, \bar{Y}):-\bar{X} \tan \alpha<\bar{Y}<0, \bar{X}>0\} .
$$

The linear harmonic problem (3.54)-(3.57) is a spectral problem with spectral parameter $k \in \mathbb{C}$. We henceforth refer to this spectral problem as $[\operatorname{SP}(k)]$. It is clear that $[\operatorname{SP}(k)]$ has the trivial solution for each $k \in \mathbb{C}$. Further, let $\bar{\Phi}_{k}^{\prime}: \mathcal{G}_{\infty} \rightarrow \mathbb{C}$ be a non-trivial solution to $[\mathrm{SP}(k)]$, then it is straightforward to establish the following.

(i) When $k \in \mathbb{R}^{+}$

$$
\begin{aligned}
\bar{\Phi}_{k}^{\prime}(\bar{R}, \theta)= & \left(c_{k} \mathrm{e}^{\mathrm{i} k \bar{R} \cos \theta}+d_{k} \mathrm{e}^{-\mathrm{i} k \bar{R} \cos \theta}\right) \mathrm{e}^{k \bar{R} \sin \theta} \\
& +a_{k} \frac{1}{\bar{R}^{\pi / 2 \alpha}} \sin \frac{\pi}{2 \alpha} \theta+O\left(\frac{1}{\bar{R}^{\pi / 2 \alpha+1}}\right),
\end{aligned}
$$

as $\bar{R} \rightarrow \infty$ uniformly for $-\alpha \leqslant \theta \leqslant 0$. Here, $c_{k}, d_{k}, a_{k} \in \mathbb{C}$ are not all zero constants.

(ii) When $k=0$

$$
\bar{\Phi}_{0}^{\prime}(\bar{R}, \theta)=a_{0}+b_{0} \frac{1}{\bar{R}^{\pi / \alpha}} \cos \frac{\pi}{\alpha} \theta+O\left(\frac{1}{\bar{R}^{2 \pi / \alpha}}\right)
$$

as $\bar{R} \rightarrow \infty$ uniformly for $-\alpha \leqslant \theta \leqslant 0$. Here $a_{0}, b_{0} \in \mathbb{C}$ are not both zero constants. (iii) When $k \in \mathbb{C} \backslash\left(\mathbb{R}^{+} \cup\{0\}\right)$

$$
\bar{\Phi}_{k}^{\prime}(\bar{R}, \theta)=a_{k} \frac{1}{\bar{R}^{\pi / 2 \alpha}} \sin \frac{\pi}{2 \alpha} \theta+O\left(\frac{1}{\bar{R}^{\pi / 2 \alpha+1}}\right),
$$

as $\bar{R} \rightarrow \infty$ uniformly for $-\alpha \leqslant \theta \leqslant 0$. Here, $a_{k} \in \mathbb{C}$ is a non-zero constant.

Our objective now is to classify the spectrum of $[\operatorname{SP}(k)]$. We define the spectrum of $[\mathrm{SP}(k)]$ to be $\boldsymbol{S}$, where

$$
S=\{k \in \mathbb{C}:[\operatorname{SP}(k)] \text { has a non-trivial solution }\} .
$$

The set of eigenvalues of $[\operatorname{SP}(k)]$ is $\boldsymbol{S}^{d}$, where

$$
S^{d}=\left\{k \in \mathbb{C}: \exists \text { a non-trivial solution to }[\mathrm{SP}(k)] \text { with } \bar{\Phi}^{\prime} \rightarrow 0 \text { as } \bar{R} \rightarrow \infty\right\},
$$

and the continuous spectrum of $[\operatorname{SP}(k)]$ is $\boldsymbol{S}^{c}$, where

$$
S^{c}=\left\{k \in \mathbb{C}: \exists \text { a non-trivial solution to }[\operatorname{SP}(k)] \text { with } \bar{\Phi}^{\prime} \nrightarrow 0 \text { as } \bar{R} \rightarrow \infty\right\},
$$

with the limits as $\bar{R} \rightarrow \infty$ considered as uniform for $-\alpha \leqslant \theta \leqslant 0$. We observe that

$$
\boldsymbol{S}=\boldsymbol{S}^{c} \cup \boldsymbol{S}^{d} .
$$


Following Needham (2012), together with the use of (3.61)-(3.63) above we can establish that (see appendix A)

$$
S^{c}=\mathbb{R}^{+} \cup\{0\}, \quad S^{d}=\varnothing,
$$

and so

$$
S=\mathbb{R}^{+} \cup\{0\} .
$$

We now introduce $S_{\lambda} \subseteq \mathbb{C}$, where

$$
\boldsymbol{S}_{\lambda}=\left\{\lambda \in \mathbb{C}: \lambda^{2}=-\mu k, k \in \boldsymbol{S}\right\} .
$$

Following (3.69), for $\mu>0$, we have

$$
\boldsymbol{S}_{\lambda}=\boldsymbol{S}_{\lambda}^{+}=\left\{\lambda \in \mathbb{C}: \lambda= \pm \mathrm{i} \sqrt{\mu} k^{1 / 2}, k \in \mathbb{R}^{+} \cup\{0\}\right\},
$$

and, for $\mu<0$, we have

$$
\boldsymbol{S}_{\lambda}=\boldsymbol{S}_{\lambda}^{-}=\left\{\lambda \in \mathbb{C}: \lambda= \pm \sqrt{(-\mu)} k^{1 / 2}, k \in \mathbb{R}^{+} \cup\{0\}\right\} .
$$

Now, the linear evolution problem (3.45)-(3.52) has a solution represented as a Fourier type integral with respect to $\lambda$ over the elementary solutions (3.53), with $\lambda \in \boldsymbol{S}_{\lambda}^{+}$ $(\mu>0)$ or $\lambda \in S_{\lambda}^{-}(\mu<0)$ and density functions chosen to satisfy the initial conditions (3.51) and (3.52). We conclude that the linear evolution problem (3.45)-(3.52) is:

(i) well-posed and stable when $\operatorname{Re}(\lambda) \geqslant 0$ for all $\lambda \in \boldsymbol{S}_{\lambda}$;

(ii) well-posed and unstable when there exists $M \in \mathbb{R}$ such that $\operatorname{Re}(\lambda) \geqslant M$ for all $\lambda \in \boldsymbol{S}_{\lambda}$, and there exists $\lambda^{*} \in \boldsymbol{S}_{\lambda}$ such that $\operatorname{Re}\left(\lambda^{*}\right)<0$;

(iii) ill-posed when there exists a sequence $\left\{\lambda_{n}\right\}_{n \in \mathbb{N}}$, with $\lambda_{n} \in S_{\lambda}$ for all $n \in \mathbb{N}$, and such that $\operatorname{Re}\left(\lambda_{n}\right) \rightarrow-\infty$ as $n \rightarrow \infty$.

It now follows directly from (3.71) and (3.72) that the linear evolution problem (3.45)-(3.52) is:

(i) well-posed and stable when $\mu>0$;

(ii) ill-posed when $\mu<0$.

We conclude that [IBVP] is well-posed and stable when $(\alpha, \mu) \in(0, \pi / 2) \times \mathbb{R}^{+}$, whilst [IBVP] is ill-posed when $(\alpha, \mu) \in(0, \pi / 2) \times \mathbb{R}^{-}$. We should note here that the time scale for ill-posed growth or stable decay for the perturbation close to the initial contact point corresponds to $\tau \gg 1$, which in the original time variable has $t \gg O\left(\delta^{\alpha / \pi}\right)$.

We next consider the problem (3.45)-(3.52) in the case $(\alpha, \mu) \in(0, \pi / 4] \times\{0\}$, where it is straightforward to show that the only solution is given by

$$
\begin{gathered}
\Phi^{\prime}(\bar{X}, \bar{Y}, \tau)=0, \quad \bar{X} \geqslant 0,-\bar{X} \tan \alpha \leqslant \bar{Y} \leqslant 0, \tau \geqslant 0, \\
H^{\prime}(\bar{X}, \tau)=\eta_{0}(\bar{X}), \quad \bar{X} \geqslant 0, \tau \geqslant 0,
\end{gathered}
$$

and thus the linear evolution problem (3.45)-(3.52) is well-posed and stable for all pairs $(\alpha, \mu) \in(0, \pi / 4] \times\{0\}$. We conclude that [IBVP] is well-posed and stable when $(\alpha, \mu) \in(0, \pi / 4] \times\{0\}$.

It remains to consider the well-posedness and stability of [IBVP] for those pairs $(\alpha, \mu) \in(\pi / 4, \pi / 2) \times \mathcal{G}(\delta)$, with $\mathcal{G}(\delta)$ being an $o(1)$ neighbourhood of $\mu=0$ as $\delta \rightarrow 0$. 
4. The case $(\alpha, \mu) \in(\pi / 4, \pi / 2) \times \mathcal{G}(\delta)$

We now investigate the well-posedness and stability of the problem [IBVP] with respect to perturbations in the initial data for those pairs $(\alpha, \mu) \in(\pi / 4, \pi / 2) \times \mathcal{G}(\delta)$, with $\mathcal{G}(\delta)$ being an $o(1)$ neighbourhood of $\mu=0$ as $\delta \rightarrow 0$. We write

$$
\mu=\varepsilon(\delta) \bar{\mu},
$$

where $\bar{\mu}=O(1)$ and $\varepsilon(\delta)=o(1)$ as $\delta \rightarrow 0$. Here the gauge function $\varepsilon(\delta)$ will be determined in the course of the analysis. Following an examination of the inner-inner region asymptotic expansions given in GNB (see (5.2) and (5.3)), we require $t^{\gamma} \sim \delta$ and $t^{\gamma} \sim \delta^{m}$, which, as $\delta \rightarrow 0$, requires

$$
t=O\left(\delta^{1 / \gamma}\right), \quad \text { with } m=1,
$$

where $\gamma=1 /(1-\pi / 4 \alpha)$. Hereafter we will refer to the initially perturbed modification of [IBVP] as [IBVP]", and we will address [IBVP]" as $\delta \rightarrow 0$ with $t=O\left(\delta^{1 / \gamma}\right)$. Thus we write

$$
t=\delta^{1 / \gamma} \tau,
$$

where $\tau=O(1)$ as $\delta \rightarrow 0$. We can now write [IBVP]" in terms of $\bar{x}, y, \phi, \eta, \tau$ and $\delta$, which becomes

$$
\begin{gathered}
\nabla^{2} \phi=0, \quad(\bar{x}, y) \in \mathcal{D}(\tau), \tau>0 \\
\nabla \phi \cdot \hat{\boldsymbol{n}}=\delta^{1 / \gamma} \tau(\varepsilon(\delta) \bar{\mu}-1) \cos \alpha, \quad y=-\bar{x} \tan \alpha, \bar{x}_{p}(\tau)<\bar{x}<\cot \alpha, \tau>0 \\
\phi_{y}=0, \quad y=-1, \bar{x}>\cot \alpha, \tau>0 \\
\eta_{\tau}+\left[\delta^{1 / \gamma} \phi_{\bar{x}}-\delta^{2 / \gamma} \tau(\varepsilon(\delta) \bar{\mu}-1) \cot \alpha\right] \eta_{\bar{x}}-\delta^{1 / \gamma} \phi_{y}=0 \\
y=\eta(\bar{x}, \tau), \bar{x}>\bar{x}_{p}(\tau), \tau>0 \\
\phi_{\tau}-\delta^{2 / \gamma} \tau(\varepsilon(\delta) \bar{\mu}-1) \cot \alpha \phi_{\bar{x}}+\delta^{1 / \gamma} \frac{1}{2}|\nabla \phi|^{2}+\delta^{1 / \gamma} \eta=0 \\
y=\eta(\bar{x}, \tau), \bar{x}>\bar{x}_{p}(\tau), \tau>0 \\
\eta+\bar{x}_{p}(\tau) \tan \alpha=0, \quad \bar{x}=\bar{x}_{p}(\tau), \tau>0 \\
\eta \rightarrow 0, \quad \text { as } \bar{x} \rightarrow \infty, \tau>0
\end{gathered}
$$

The structure of the solution to [IBVP] as $t \rightarrow 0^{+}$(see GNB) indicates that there will be three asymptotic regions in the solution to [IBVP]" as $\delta \rightarrow 0$ when $\tau=O(1)$. In the outer region we have $(\bar{x}, y) \in \overline{\mathcal{D}}(\tau)=O(1)$ as $\delta \rightarrow 0$ when $\tau=O(1)$, and we consider this first.

\subsection{Outer region for $[I B V P]^{\prime \prime}$ when $\tau=O(1)$ as $\delta \rightarrow 0$}

We begin in an outer region in which $(\bar{x}, y) \in \overline{\mathcal{D}}(\tau)=O(1)$, when $\tau=O(1)$ as $\delta \rightarrow 0$, after which we will require two additional regions, in which $(\bar{x}, y)=o(1)$ as $\delta \rightarrow 0$, in order to capture the initial interaction between the plate and the free surface. We observe that conditions (4.5) and (4.7) require $\phi=O\left(\delta^{1 / \gamma}\right)$ and $\eta=O\left(\delta^{2 / \gamma}\right)$ as $\delta \rightarrow 0$ in the outer region. Thus we introduce the asymptotic expansions

$$
\begin{gathered}
\phi(\bar{x}, y, \tau)=-\delta^{1 / \gamma} \cos \alpha \phi^{\prime \prime}(\bar{x}, y, \tau)+O\left(\delta^{2 / \gamma}\right), \\
\eta(\bar{x}, \tau)=\delta^{2 / \gamma} \eta^{\prime \prime}(\bar{x}, \tau)+O\left(\delta^{3 / \gamma}\right)
\end{gathered}
$$


as $\delta \rightarrow 0$ in the outer region, with the factor $-\cos \alpha$ in (4.14) included for algebraic convenience at a later stage. Substituting the expansions (4.14) and (4.15) into [IBVP]" we obtain, at leading order, a problem equivalent to (3.15)-(3.22) in the case $\mu=0$. It follows that, after writing

$$
\phi^{\prime \prime}(\bar{x}, y, \tau)=\tau \bar{\phi}^{\prime \prime}(\bar{x}, y), \quad \eta^{\prime \prime}(\bar{x}, \tau)=\tau^{2} \bar{\eta}^{\prime \prime}(\bar{x}),
$$

then $\bar{\phi}^{\prime \prime}$ and $\bar{\eta}^{\prime \prime}$ are given (for some real constants $A_{n}, B_{n}$ and $C_{n}$ ) by (3.24)-(3.28). The form of $\bar{\phi}^{\prime \prime}$ and $\bar{\eta}^{\prime \prime}$ as $(\bar{x}, y) \rightarrow(0,0)$ is given by (3.29) and (3.30), where we again have singular behaviour as $(\bar{x}, y) \rightarrow(0,0)$, which motivates the introduction of an inner region in which $(\bar{x}, y)=o(1)$ as $\delta \rightarrow 0$.

\subsection{Inner region for $[I B V P]^{\prime \prime}$ when $\tau=O(1)$ as $\delta \rightarrow 0$}

We set $(\bar{x}, y)=O(\bar{v}(\delta))$ with $\bar{v}(\delta)=o(1)$ as $\delta \rightarrow 0$ in the inner region. It follows from (4.15), and (4.16), along with (3.23) and (3.30), that $\eta=O\left(\delta^{2 / \gamma}\right)$ as $\delta \rightarrow 0$ in the inner region, and so, in order to capture the free surface in the inner region, we must take $\bar{v}(\delta)=O\left(\delta^{2 / \gamma}\right)$ as $\delta \rightarrow 0$; thus without loss of generality, we set $\bar{v}(\delta)=\delta^{2 / \gamma}$. An examination of (4.14), together with (3.23) and (3.29), then requires that $\phi=O\left(\delta^{3 / \gamma}\right)$ as $\delta \rightarrow 0$ in the inner region. Thus we introduce scaled inner region coordinates $(X, Y)$ by

$$
\bar{x}=\delta^{2 / \gamma} X, \quad y=\delta^{2 / \gamma} Y,
$$

with $(X, Y)=O(1)$ as $\delta \rightarrow 0$ in the inner region $(\dagger)$. The location of the plate in the inner region is given by $Y=-X \tan \alpha$, whilst the contact point is denoted by $(X, Y)=\left(X_{p}(\tau), Y_{p}(\tau)\right)$, where $\bar{x}_{p}(\tau)=\delta^{2 / \gamma} X_{p}(\tau)$ and $y_{p}(\tau)=\delta^{2 / \gamma} Y_{p}(\tau)(\dagger)$. We now write the free surface and velocity potential in the inner region as

$$
\begin{gathered}
\eta(X, \tau)=\delta^{2 / \gamma} \eta_{I}(X, \tau), \quad X \geqslant X_{p}(\tau), \tau \geqslant 0 ; \\
\phi(X, Y, \tau)=\delta^{3 / \gamma} \phi_{I}(X, Y, \tau), \quad X \geqslant X_{p}(\tau),-X \tan \alpha \leqslant Y \leqslant \eta_{I}(X, \tau), \tau \geqslant 0 ;
\end{gathered}
$$

where $\eta_{I}(X, \tau), \phi_{I}(X, Y, \tau)=O(1)$ as $\delta \rightarrow 0(\dagger)$. The inner region expansions are then introduced as

$$
\begin{gathered}
\eta_{I}(X, \tau)=\widetilde{\eta}_{0}^{\prime \prime}(X, \tau)+\delta^{(\pi / \alpha-2) / \gamma} \widetilde{\eta}_{1}^{\prime \prime}(X, \tau)+o\left(\delta^{(\pi / \alpha-2) / \gamma}\right), \\
\phi_{I}(X, Y, \tau)=\widetilde{\phi}_{0}^{\prime \prime}(X, Y, \tau)+\delta^{(\pi / \alpha-2) / \gamma} \widetilde{\phi}_{1}^{\prime \prime}(X, Y, \tau)+o\left(\delta^{(\pi / \alpha-2) / \gamma}\right),
\end{gathered}
$$

as $\delta \rightarrow 0$ where $(X, Y)=O(1)$, with the form of the correction terms having been deduced from (4.17), together with (3.14), (3.23) and (3.30). It also follows from (4.17) and (4.20) that the free surface in the inner region is located at

$$
Y=\widetilde{\eta}_{0}^{\prime \prime}(X, \tau)+\delta^{(\pi / \alpha-2) / \gamma} \widetilde{\eta}_{1}^{\prime \prime}(X, \tau)+o\left(\delta^{(\pi / \alpha-2) / \gamma}\right), \quad X>X_{p}(\tau), \tau \geqslant 0,
$$

and hence we must expand

$$
X_{p}(\tau)=X_{0}(\tau)+\delta^{(\pi / \alpha-2) / \gamma} X_{1}(\tau)+o\left(\delta^{(\pi / \alpha-2) / \gamma}\right), \quad \tau \geqslant 0,
$$

as $\delta \rightarrow 0$, after which $Y_{p}(\tau)=-X_{p}(\tau) \tan \alpha(\dagger)$.

We can now write [IBVP]" in terms of the inner variables and substitute from (4.20) and (4.21). The problem obtained at leading order, when supplemented with matching 
conditions to the outer region (via Van Dyke's matching principle, (Van Dyke 1964)), has the solution

$$
\begin{gathered}
\widetilde{\phi}_{0}^{\prime \prime}(X, Y, \tau)=-\tau Y-\frac{1}{6} \tau^{2}, \quad X \geqslant X_{0}(\tau),-X \tan \alpha \leqslant Y \leqslant-\frac{1}{2} \tau^{2}, \tau \geqslant 0 \\
\widetilde{\eta}_{0}^{\prime \prime}(X, \tau)=-\frac{1}{2} \tau^{2}, \quad X \geqslant X_{0}(\tau), \tau \geqslant 0 ; \\
X_{0}(\tau)=\frac{1}{2} \tau^{2} \cot \alpha, \quad \tau \geqslant 0 .
\end{gathered}
$$

It follows from (4.24) and (4.25), using (4.17)-(4.21), that the regularity conditions (see GNB (2.16)) are satisfied at leading order in the inner region. We now formulate the problem at $O\left(\delta^{(\pi / \alpha-2) / \gamma}\right)$, where it is convenient to introduce the coordinates $(\bar{X}, \bar{Y})$ according to

$$
X=\frac{1}{2} \tau^{2} \cot \alpha+\bar{X}, \quad Y=-\frac{1}{2} \tau^{2}+\bar{Y},
$$

which is simply a shift of origin from the original inner region coordinates $(X, Y)(\dagger)$. We now obtain the problem for $\widetilde{\phi}_{1}^{\prime \prime}, \widetilde{\eta}_{1}^{\prime \prime}$ and $X_{1}$, as

$$
\begin{gathered}
\bar{\nabla}^{2} \widetilde{\phi}_{1}^{\prime \prime}=0, \quad \bar{X}>0,-\bar{X} \tan \alpha<\bar{Y}<0, \tau>0 ; \\
\bar{\nabla} \widetilde{\phi}_{1}^{\prime \prime} \cdot \hat{\boldsymbol{n}}=0, \quad \bar{X}>0, \bar{Y}=-\bar{X} \tan \alpha, \tau>0 ; \\
\widetilde{\eta}_{1, \tau}^{\prime \prime}-\widetilde{\phi}_{1, \bar{Y}}^{\prime \prime}=0, \quad \bar{X}>0, \bar{Y}=0, \tau>0 ; \\
\widetilde{\phi}_{1, \tau}^{\prime \prime}=0, \quad \bar{X}>0, \bar{Y}=0, \tau>0 ; \\
\widetilde{\phi}_{1}^{\prime \prime}(\bar{R}, \theta)=\tau A_{0}(\alpha) \cos \alpha \bar{R}^{\pi / 2 \alpha} \cos \frac{\pi}{\alpha}(\theta+\alpha)+o\left(\bar{R}^{\pi / 2 \alpha}\right) \\
\text { as } \bar{R} \rightarrow \infty,-\alpha<\theta<0, \tau>0 ; \\
\widetilde{\eta}_{1}^{\prime \prime}(\bar{X})=-\tau^{2} A_{0}(\alpha) \frac{\pi}{4 \alpha} \bar{X}^{\pi / 2 \alpha-1}+o\left(\bar{X}^{\pi / 2 \alpha-1}\right) \quad \text { as } \bar{X} \rightarrow \infty, \tau>0 ; \\
\widetilde{\phi}_{1}^{\prime \prime}(\bar{X}, \bar{Y}, 0)=0, \quad \bar{X} \geqslant 0,-\bar{X} \tan \alpha \leqslant \bar{Y} \leqslant 0 ; \\
\widetilde{\eta}_{1}^{\prime \prime}(\bar{X}, 0)=0, \quad \bar{X} \geqslant 0 .
\end{gathered}
$$

Here $\bar{\nabla}=(\partial / \partial \bar{X}, \partial / \partial \bar{Y}),(4.32)$ and (4.33) are the matching conditions with the outer region, $A_{0}(\alpha)(<0)$ is given in GNB (see (3.10)), and we have introduced polar coordinates $(\bar{R}, \theta)$, given by $\bar{X}=\bar{R} \cos \theta, \bar{Y}=\bar{R} \sin \theta(\dagger)$. Additionally we have

$$
X_{1}(\tau)=-\widetilde{\eta}_{1}^{\prime \prime}(0, \tau) \cot \alpha, \quad \tau>0 .
$$

It is straightforward to show that, in this degenerate case, the solution to the problem (4.28)-(4.35), which has least singular behaviour at $(\bar{X}, \bar{Y})=(0,0)$ is simply given exactly by the far-field functions, that is

$$
\begin{gathered}
\widetilde{\phi}_{1}^{\prime \prime}(\bar{R}, \theta, \tau)=\tau A_{0}(\alpha) \cos \alpha \bar{R}^{\pi / 2 \alpha} \cos \frac{\pi}{2 \alpha}(\theta+\alpha), \\
\bar{R}>0,-\alpha<\theta<0, \tau>0 ; \\
\widetilde{\eta}_{1}^{\prime \prime}(\bar{X}, \tau)=-\tau^{2} A_{0}(\alpha) \frac{\pi}{4 \alpha} \cos \alpha \bar{X}^{\pi / 2 \alpha-1}, \quad \bar{X}>0, \tau>0 .
\end{gathered}
$$

We see from (4.37) and (4.38) that a weak singularity in derivatives (that is $\widetilde{\eta}_{1, \bar{X}}^{\prime \prime}$, and $\left.\bar{\nabla} \widetilde{\phi}_{1}^{\prime \prime}\right)$ persists close to the contact point and, in particular, when $(\bar{X}, \bar{Y})=O\left(\delta^{(\pi / \alpha-2) / \gamma}\right)$. This requires the introduction of an inner-inner region, in which $(\bar{X}, \bar{Y})=o(1)$ as $\delta \rightarrow 0$ and within which the full regularity conditions at the contact point are satisfied. 


\subsection{Inner-inner region for [IBVP]" when $\tau=O(1)$ as $\delta \rightarrow 0$}

We set $(\bar{X}, \bar{Y})=O(\Delta(\delta))$, with $\Delta(\delta)=O(1)$ as $\delta \rightarrow 0$. It then follows from (4.20), (4.25) and (4.38) that the free surface is located at $\bar{Y}=\tau^{2} / 2+\eta_{I}=O\left(\delta^{(\pi / \alpha-2) / \gamma} \Delta(\delta)^{\pi / 2 \alpha-1}\right)$ as $\delta \rightarrow 0$ in the inner-inner region. Therefore, to capture the free surface in the inner-inner region, we must take $\Delta(\delta)=O\left(\delta^{\pi / 2 \alpha-1}\right)$, and so, without loss of generality, we set $\Delta(\delta)=\delta^{\pi / 2 \alpha-1}$. We introduce scaled inner-inner region coordinates $(\widetilde{x}, \widetilde{y})$ by

$$
\bar{X}=\delta^{\pi / 2 \alpha-1} \widetilde{x}, \quad \bar{Y}=\delta^{\pi / 2 \alpha-1} \widetilde{y},
$$

where $(\widetilde{x}, \widetilde{y})=O(1)$ as $\delta \rightarrow 0$ in the inner-inner region $(\dagger)$. The location of the plate in the inner-inner region is given by $\tilde{y}=-\widetilde{x} \tan \alpha$, with the plate and contact point denoted by $(\widetilde{x}, \widetilde{y})=\left(\widetilde{x}_{p}(\tau), \widetilde{y}_{p}(\tau)\right)$, with $\widetilde{x}_{p}(\tau)=\delta^{\pi / 2 \alpha-1} \bar{X}_{p}(\tau)$ and $\widetilde{y}_{p}(\tau)=\delta^{\pi / 2 \alpha-1} \bar{Y}_{p}(\tau)$ $(\dagger)$, where

$$
\bar{X}_{p}(\tau)=X_{p}(\tau)-\frac{1}{2} \tau^{2} \cot \alpha, \quad \bar{Y}_{p}(\tau)=Y_{p}(\tau)+\frac{1}{2} \tau^{2} .
$$

An examination of (4.20), (4.21), (4.24), (4.25), (4.37) and (4.38) requires that $\eta_{I}=$ $-\tau^{2} / 2+O\left(\delta^{\pi / 2 \alpha-1}\right)$ and $\phi_{I}=\tau^{3} / 3-\delta^{\pi / 2 \alpha-1} \tau \widetilde{y}+O\left(\delta^{\pi / \alpha-2}\right)$ as $\delta \rightarrow 0$. We thus write the free surface and velocity potential in the inner-inner region as

$$
\begin{gathered}
\eta_{I}(\widetilde{x}, \tau)=-\frac{1}{2} \tau^{2}+\delta^{\pi / 2 \alpha-1} \eta_{I I}(\widetilde{x}, \tau), \quad \widetilde{x}>\widetilde{x}_{p}(\tau), \tau \geqslant 0 ; \\
\begin{array}{r}
\phi_{I}(\widetilde{x}, \widetilde{y}, \tau)=\frac{1}{3} \tau^{3}-\delta^{\pi / 2 \alpha-1} \tau \widetilde{y}+\delta^{\pi / \alpha-2} \phi_{I I}(\widetilde{x}, \widetilde{y}, \tau), \\
\tilde{x} \geqslant \widetilde{x}_{p}(\tau),-\widetilde{x} \tan \alpha \leqslant \widetilde{y} \leqslant \eta_{I I}(\widetilde{x}, \tau), \tau \geqslant 0 ;
\end{array}
\end{gathered}
$$

where $\eta_{I I}, \phi_{I I}=O(1)$ as $\delta \rightarrow 0(\dagger)$. The inner-inner region asymptotic expansions are then introduced as

$$
\eta_{I I}(\widetilde{x}, \tau)=\widehat{\eta}_{0}^{\prime \prime}(\widetilde{x}, \tau)+o(1), \quad \phi_{I I}(\widetilde{x}, \widetilde{y}, \tau)=\widehat{\phi}_{0}^{\prime \prime}(\widetilde{x}, \widetilde{y}, \tau)+o(1),
$$

as $\delta \rightarrow 0$, where $(\widetilde{x}, \widetilde{y})=O(1)$. It follows from (4.39) and (4.43) that the free surface in the inner-inner region is located at

$$
\widetilde{y}(\tau)=\widehat{\eta}_{0}^{\prime \prime}(\widetilde{x}, \tau)+o(1), \quad \widetilde{x}>\widetilde{x}_{p}(\tau), \tau \geqslant 0,
$$

and hence we must expand

$$
\widetilde{x}_{p}(\tau)=\widetilde{x}_{0}(\tau)+o(1), \quad \tau \geqslant 0,
$$

as $\delta \rightarrow 0$, after which $\widetilde{y}_{p}(\tau)=-\widetilde{x}_{p}(\tau) \tan \alpha(\dagger)$. An examination of the plate boundary condition (4.5), (4.39) and (4.42), together with (4.1), then requires that the gauge function $\varepsilon(\delta)=O\left(\delta^{\pi / 2 \alpha-1}\right)$ (from which it follows that $\mathcal{G}(\varepsilon)=O\left(\delta^{\pi / 2 \alpha-1}\right)$ ), and so, without loss of generality, we set $\varepsilon(\delta)=\delta^{\pi / 2 \alpha-1}$. We are now able to write [IBVP]" in terms of the inner-inner region variables and substitute from (4.43). It is convenient to first introduce the dependent variables $\Phi^{\prime \prime}$ and $H^{\prime \prime}$, according to

$$
\left.\widehat{\phi}_{0}^{\prime \prime}(\widetilde{x}, \widetilde{y}, \tau)=\Phi^{\prime \prime}(\widetilde{x}, \widetilde{y}, \tau)+\bar{\mu} \tau \widetilde{y}-\frac{1}{3} \bar{\mu}^{2} \tau^{3}, \quad \widehat{\eta}_{0}^{\prime \prime} \widetilde{x}, \tau\right)=H^{\prime \prime}(\widetilde{x}, \tau)+\frac{1}{2} \bar{\mu} \tau^{2}
$$

along with the translated coordinates $(\widehat{x}, \widehat{y})$, according to

$$
\widehat{x}=\tilde{x}+\frac{1}{2} \bar{\mu} \tau^{2} \cot \alpha, \quad \widehat{y}=\tilde{y}-\frac{1}{2} \bar{\mu} \tau^{2},
$$


which is simply a shift of origin from the original inner-inner coordinates $(\widetilde{x}, \widetilde{y})(\dagger)$. The variable changes (4.46) and (4.47) have been chosen for algebraic convenience at a later stage. We obtain at leading order the following nonlinear harmonic evolution free boundary problem for $\Phi^{\prime \prime}, H^{\prime \prime}$ and $\widehat{x}_{0}$, namely

$$
\begin{gathered}
\widehat{\nabla}^{2} \Phi^{\prime \prime}=0, \quad \widehat{x}>\widehat{x}_{0}(\tau),-\widehat{x} \tan \alpha<\widehat{y}<H^{\prime \prime}(\widehat{x}, \tau), \tau>0 ; \\
\widehat{\nabla} \Phi^{\prime \prime} \cdot \hat{\boldsymbol{n}}=0, \quad \widehat{x}>\widehat{x}_{0}(\tau), \widehat{y}=-\widehat{x} \tan \alpha, \tau>0 ; \\
H_{\tau}^{\prime \prime}+\Phi_{\widehat{x}}^{\prime \prime} H_{\widehat{x}}^{\prime \prime}-\Phi_{\widehat{y}}^{\prime \prime}=0, \quad \widehat{x}>\widehat{x}_{0}(\tau), \widehat{y}=H^{\prime \prime}(\widehat{x}, \tau), \tau>0 ; \\
\Phi_{\tau}^{\prime \prime}+\frac{1}{2}\left|\widehat{\nabla} \Phi^{\prime \prime}\right|^{2}+\bar{\mu} H^{\prime \prime}=0, \quad \widehat{x}>\widehat{x}_{0}(\tau), \widehat{y}=H^{\prime \prime}(\widehat{x}, \tau), \tau>0 ; \\
H^{\prime \prime}\left(\widehat{x}_{0}(\tau), \tau\right)=-\widehat{x}_{0}(\tau) \tan \alpha, \quad \tau>0 ; \\
\Phi^{\prime \prime}(\widehat{x}, \widehat{y}, 0)=0, \quad \widehat{x}>0,-\widehat{x} \tan \alpha<\widehat{y}<\eta_{0}(\widehat{x}) ; \\
H^{\prime \prime}(\widehat{x}, 0)=\eta_{0}(\widehat{x}), \quad \widehat{x}>0 ; \\
\Phi^{\prime \prime}(\widehat{r}, \theta, \tau)=\tau A_{0}(\alpha) \cos \alpha \widehat{r}^{\pi / 2 \alpha} \cos \frac{\pi}{2 \alpha}(\theta+\alpha)+o\left(\widehat{r}^{\pi / 2 \alpha}\right) \\
\operatorname{as} \widehat{r} \rightarrow \infty,-\alpha<\theta<0, \tau>0 ; \\
H^{\prime \prime}(\widehat{x}, \tau)=-\tau^{2} A_{0}(\alpha) \frac{\pi}{4 \alpha} \cos \alpha \widehat{x}^{\pi / 2 \alpha-1}+o\left(\widehat{x}^{\pi / 2 \alpha-1}\right) \quad \text { as } \widehat{x} \rightarrow \infty, \tau>0 ;
\end{gathered}
$$

where

$$
\widehat{x}_{0}(\tau)=\widetilde{x}_{0}(\tau)+\frac{1}{2} \bar{\mu} \tau^{2} \cot \alpha .
$$

Here $\widehat{\nabla}=(\partial / \partial \widehat{x}, \partial / \partial \widehat{y}), A_{0}(\alpha)(<0)$ is as given in GNB (see (3.10)), and we have introduced polar coordinates $(\widehat{r}, \theta)$ as $\widehat{x}=\widehat{r} \cos \theta, \widehat{y}=\widehat{r} \sin \theta(\dagger)$. The initial boundary value problem (4.48)-(4.56), henceforth referred to as [EBVP], can now be solved numerically using a boundary integral method, which follows the approach discussed in GNB, with implicit time stepping to evolve the solution in time $\tau$.

The first thing to observe concerning [EBVP], is that in the case when $\eta_{0}(\widehat{x})=0$ for $\hat{x} \geqslant 0$, then the solution to [EBVP] has the similarity structure

$$
\begin{gathered}
\Phi^{\prime \prime}(\widehat{x}, \widehat{y}, \tau)=\tau^{\Gamma} \widehat{\Phi}^{\prime \prime}\left(\frac{\widehat{x}}{\tau^{\Gamma}}, \frac{\widehat{y}}{\tau^{\Gamma}}\right), \quad \widehat{x}>\widehat{c} \tau^{\Gamma},-\widehat{x} \tan \alpha<\widehat{y}<\tau^{\Gamma} \widehat{H}^{\prime \prime}\left(\frac{\widehat{x}}{\tau^{\Gamma}}\right), \tau>0 ; \\
H^{\prime \prime}(\widehat{x}, \tau)=\tau^{\Gamma} \widehat{H}^{\prime \prime}\left(\frac{\widehat{x}}{\tau^{\Gamma}}\right), \quad \widehat{x}>\widehat{c} \tau^{\Gamma}, \tau>0 \\
\widehat{x}_{0}(\tau)=\widehat{c} \tau^{\Gamma}, \quad \tau>0
\end{gathered}
$$

where $\widehat{c}$ is the constant satisfying $\widehat{H}^{\prime \prime}(\widehat{c})+\widehat{c}=0$ and $\Gamma$ as in GNB (see (5.1)). This is anticipated from, and conforms with, the details in GNB $(\S 5)$. We now proceed with the numerical solution of [EBVP].

In solving [EBVP] numerically we must specify the initial free surface profile. For the following results we have taken $H^{\prime \prime}(\widehat{x}, 0)$ as

$$
H^{\prime \prime}(\widehat{x}, 0)=\eta_{0}(\widehat{x})= \begin{cases}0.02(1-\cos 2 \pi \widehat{x}), & 0 \leqslant \widehat{x} \leqslant 1 \\ 0, & \widehat{x}>1\end{cases}
$$




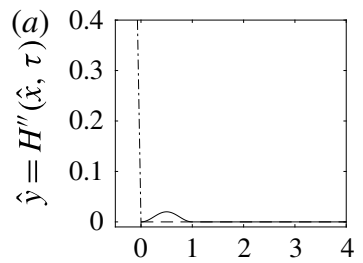

(b)

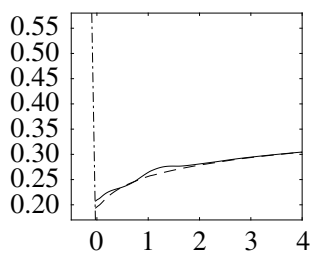

(e)
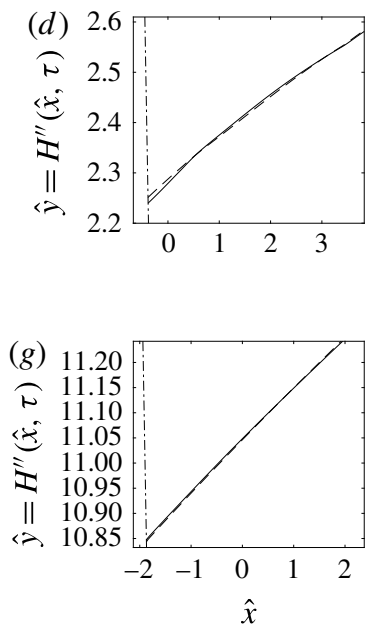

(c)

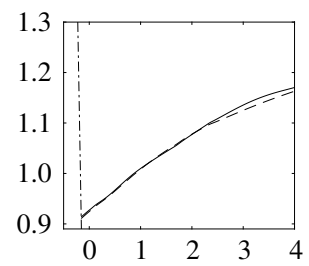

(f)

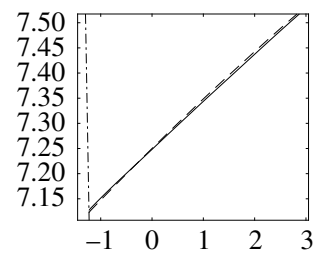

(i)

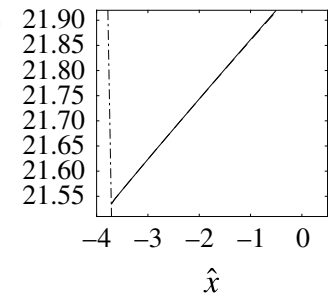

FIGURE 2. Graph of the evolution of $H^{\prime \prime}(\widehat{x}, \tau)$ (related to the free surface displacement through (4.46), (4.43), (4.41) and (4.18)) against $\hat{x}$ for the numerical solution of [EBVP], for $\tau=0, \tau=0.75, \tau=1.5, \tau=2.25, \tau=3, \tau=3.75, \tau=4.5, \tau=5.25$ and $\tau=6,(a-i)$ where $\bar{\mu}=1$ and $\alpha=1.4$. In each plot a dash-dot line shows the location of the plate, a dotted line shows the solution for the case of zero initial data and a solid line shows the solution when the initial data are as given in (4.61).

We note that the original free surface displacement is then given by

$$
\eta=-\frac{1}{2} \delta^{2 / \gamma} \tau^{2}+\delta\left(H^{\prime \prime}+\frac{1}{2} \bar{\mu} \tau^{2}\right)+o(\delta) \quad \text { as } \delta \rightarrow 0 .
$$

Numerical solutions of [EBVP] are plotted in figures 2-5. In figure 2 we present the comparison between the free surface $H^{\prime \prime}(\widehat{x}, \tau)$ calculated with zero initial data (shown in each plot as a dotted line) and the free surface $H^{\prime \prime}(\widehat{x}, \tau)$ calculated with the initial free surface profile given in (4.61) (shown in each plot as a solid line) for the case $\alpha=1.4$, with $\bar{\mu}=1$. It is clear to see, as may be anticipated from $\S 3.2$, that, when initially perturbed, the free surface $H^{\prime \prime}(\widehat{x}, \tau)$ collapses to the free surface corresponding to zero perturbation (which has the similarity solution (4.59)) as $\tau \rightarrow \infty$, indicating that the problem [EBVP] is well-posed and stable in this case. This behaviour is typical of all pairs $(\alpha, \bar{\mu})$ tested in the range $(\alpha, \bar{\mu}) \in(\pi / 4, \pi / 2) \times \mathbb{R}^{+}$, and of all initial free surface profiles $\eta_{0}(\widehat{x})$ tested. As we decrease $\bar{\mu}$ and take values with $\bar{\mu}<0$ we are unable to obtain numerically any converged solutions to [EBVP]. This indicates that the problem [EBVP] is ill-posed, which may also be anticipated from the theory presented in $\$ 3.2$. In figure 3 we present the comparison between the free surface $H^{\prime \prime}(\widehat{x}, \tau)$ calculated with zero initial data (shown in each plot as a dotted line) and the free surface $H^{\prime \prime}(\widehat{x}, \tau)$ calculated with the initial free surface profile given in (4.61) 


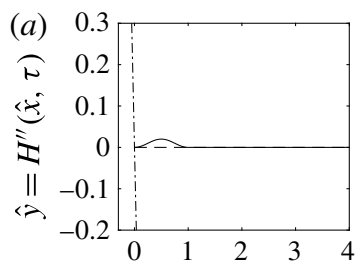

(b)

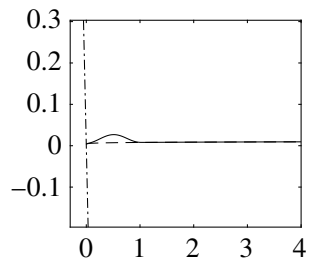

(e)

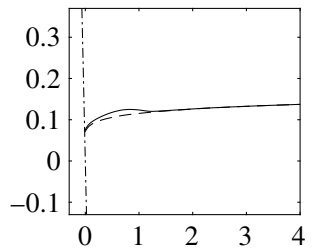

(h)
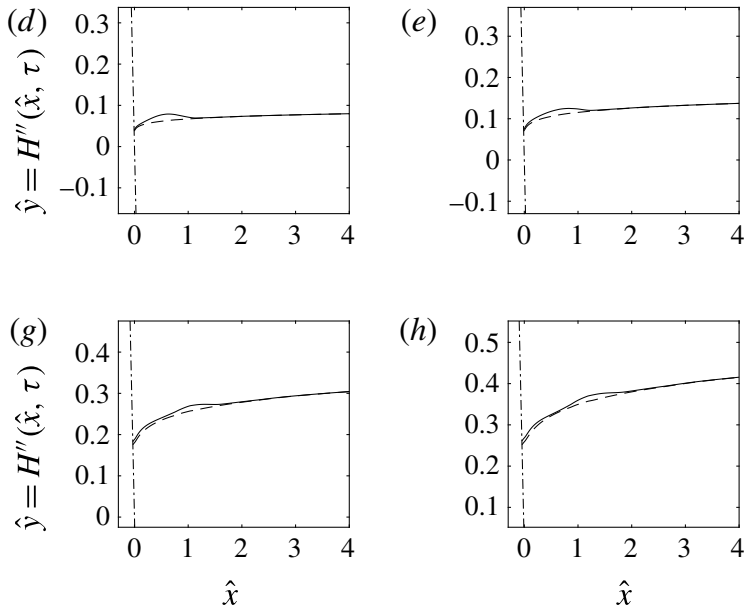

(c)

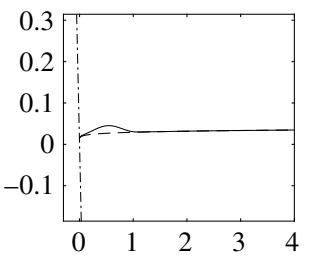

(f)

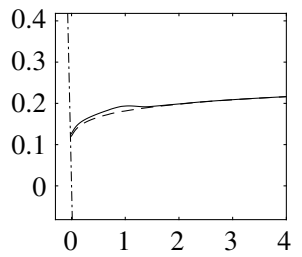

(i)

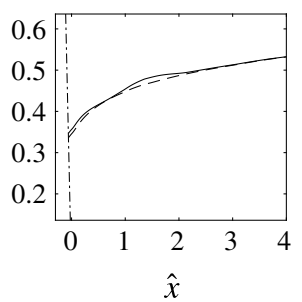

FIGURE 3. Graph of the evolution of $H^{\prime \prime}(\widehat{x}, \tau)$ (related to the free surface displacement through (4.46), (4.43), (4.41) and (4.18)) against $\hat{x}$ for the numerical solution of [EBVP], for $\tau=0, \tau=0.13, \tau=0.25, \tau=0.38, \tau=0.5, \tau=0.63, \tau=0.75, \tau=0.88$ and $\tau=1$, $(a-i)$ where $\bar{\mu}=0$ and $\alpha=1.4$. In each plot a dash-dot line shows the location of the plate, a dotted line shows the solution for the case of zero initial data and a solid line shows the solution when the initial data are as given in (4.61).

(shown in each plot as a solid line) for the case $\alpha=1.4$, with $\bar{\mu}=0$. The case $\bar{\mu}=0$ separates the regions on the $(\alpha, \mu)$ plane where the problem [EBVP] is well-posed and stable $(\bar{\mu}>0)$ from ill-posed $(\bar{\mu}<0)$, with $\mu=0$ falling into the well-posed and stable case. Finally, figures 4 and 5 demonstrate the agreement of the numerical solution with the far-field asymptotic form in [EBVP] (4.56), at least to the graphical scales shown.

\section{Conclusion}

In this paper we have studied the well-posedness and stability of the problem [IBVP]. In particular, concerning the early stage motion close to the contact point, which was examined in detail in GNB, with respect to perturbations of amplitude $\delta \ll 1$ in initial data, located in the innermost asymptotic region of the solution to [IBVP] as $t \rightarrow 0^{+}$, for each pair $(\alpha, \mu) \in(0, \pi / 2) \times \mathbb{R}$, where $\mu=1+\sigma \tan \alpha$. This has enabled us to draw the following conclusions.

(i) $(\alpha, \mu) \in(0, \pi / 2) \times \overline{\mathbb{R}}^{+}$

Here, the initial boundary value problem [IBVP] is well-posed and stable with respect to perturbations in the initial data located in the inner region of the solution to [IBVP]. The time scale for decay is $t \gg O\left(\delta^{\alpha / \pi}\right)$. 


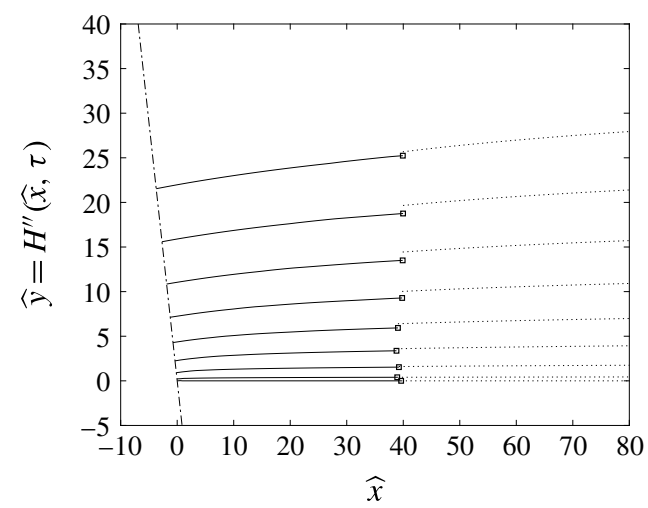

FIGURE 4. Graph of the evolution of $H^{\prime \prime}(\widehat{x}, \tau)$ (related to the free surface displacement through (4.46), (4.43), (4.41) and (4.18)) against $\hat{x}$, showing agreement with the far-field asymptotic form (4.56) for the numerical solution of [EBVP], for $\tau=0, \tau=0.75, \tau=1.5$, $\tau=2.25, \tau=3, \tau=3.75, \tau=4.5, \tau=5.25$ and $\tau=6$, where $\bar{\mu}=1$, and $\alpha=1.4$. Here $\tau$ increases reading from the bottom of the figure to the top. The dash-dot line shows the location of the plate, solid lines show the numerical solution when the initial data are as given in (4.61), and dotted lines plot the far-field asymptotic form (4.56).

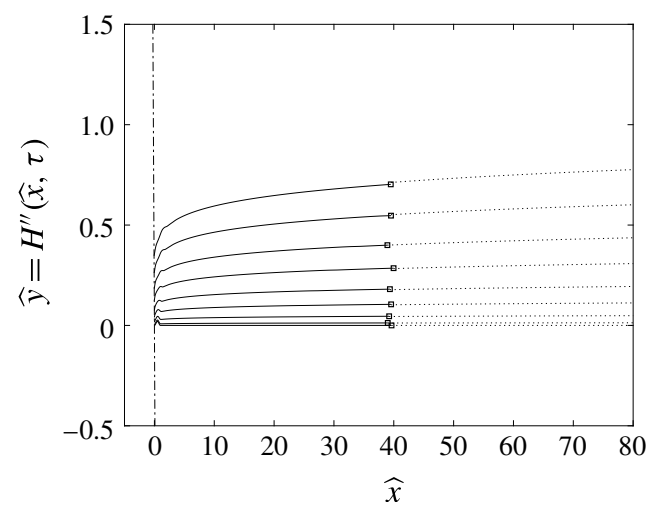

FIGURE 5. Graph of the evolution of $H^{\prime \prime}(\widehat{x}, \tau)$ (related to the free surface displacement through (4.46), (4.43), (4.41) and (4.18)) against $\widehat{x}$, showing agreement with the far-field asymptotic form (4.56) for the numerical solution of [EBVP], for $\tau=0, \tau=0.13, \tau=0.25$, $\tau=0.38, \tau=0.5, \tau=0.63, \tau=0.75, \tau=0.88$ and $\tau=1$, where $\bar{\mu}=0$ and $\alpha=1.4$. Here $\tau$ increases reading from the bottom of the figure to the top. The dash-dot line shows the location of the plate, solid lines show the numerical solution when the initial data are as given in (4.61), and dotted lines plot the far-field asymptotic form (4.56).

(ii) $(\alpha, \mu) \in(0, \pi / 2) \times \mathbb{R}^{-}$

Here, the initial boundary value problem [IBVP] is ill-posed with respect to perturbations in initial data located in the inner region of the solution to [IBVP] with the time scale for the growth due to ill-posedness being $t \gg O\left(\delta^{\alpha / \pi}\right)$.

In the above case (ii), when the solution to the unperturbed problem [IBVP] is ill-posed with respect to initial perturbations localised close to the contact point, we anticipate that the inclusion of weak surface tension effects in the model, leading 
to the problems [IBVP], [IBVP]' and [IBVP]", will restore well-posedness, with the problem [IBVP] then becoming well-posed but unstable. The physical source of the ill-posedness of the problem [IBVP], is revealed on making the following observation. Returning to GNB (see $\S \S 4$ and 5) we observe in the innermost regions that the leading-order displacement of the free surface has

$$
\eta(X, t) \sim \frac{1}{2} \sigma t^{2} \tan \alpha, \quad X>-\frac{1}{2} \sigma,
$$

when $O\left(\delta^{\alpha / \pi}\right) \ll t \ll 1$. This represents a horizontal free surface accelerating vertically downwards, with dimensionless uniform acceleration of $-\sigma \tan \alpha$. With the dimensionless acceleration due to gravity on this localised block of fluid being unity, the situation is analogous to a local Rayleigh-Taylor problem (see, for example, Sharp 1984), which (in the absence of surface tension effects) would predict the horizontal free surface to be well-posed and stable to perturbations when $1>-\sigma \tan \alpha$, but ill-posed when $1<-\sigma \tan \alpha$. Recalling that $\mu=1+\sigma \tan \alpha$, this precisely accords with the cases above emerging from the detailed theory. This further supports our contention that the inclusion of weak surface tension effects will restore well-posedness in case (ii). In a more general context, we should note that, in both parts I and II of this pair of papers, the inner regions are driven principally by the matching conditions with the outer region, and in particular through the constant $A_{0}(\alpha)$. Thus, the local flow structure, close to the initial contact point, in the early stages, will be the same for more general shaped surface piercing bodies which advance or retreat into an expanse of initially stationary fluid under gravity. Here $\alpha$ will represent the body slope at the initial contact point when motion is initiated, and the detailed body shape is simply encompassed in the value of $A_{0}(\alpha)$.

\section{Acknowledgements}

M.T.G. gratefully acknowledges financial support from EPSRC grant EP/H007830/1. We should like to thank all the referees for their detailed reading of the paper. Their comments have enabled us to significantly improve the paper.

\section{Appendix A. Classification of the spectrum of $[\operatorname{SP}(k)]$}

This appendix details the classification of the spectrum of $[\operatorname{SP}(k)]$. In $\S 3.2$ we define the spectral problem $[\mathrm{SP}(k)]$ to be given by

$$
\begin{gathered}
\bar{\nabla}^{2} \bar{\Phi}^{\prime}=0, \quad \bar{X}>0,-\bar{X} \tan \alpha<\bar{Y}<0 ; \\
\bar{\nabla} \cdot \hat{\Phi}=0, \quad \bar{X}>0, \bar{Y}=-\bar{X} \tan \alpha ; \\
\bar{\Phi}_{\bar{Y}}^{\prime}-k \bar{\Phi}^{\prime}=0, \quad \bar{X}>0, \bar{Y}=0 ; \\
\bar{\Phi}^{\prime}, \bar{\nabla} \bar{\Phi} \quad \text { bounded as } \bar{R} \rightarrow \infty, \text { uniformly for }-\alpha<\theta<0 ;
\end{gathered}
$$

with $k=-\lambda^{2} / \mu$ (for $\mu \neq 0$ ), and we require that solutions to $[\operatorname{SP}(k)], \bar{\Phi}^{\prime}: \overline{\mathcal{G}}_{\infty} \rightarrow \mathbb{C}$, have regularity given by (3.59). We define the spectrum of $[\operatorname{SP}(k)]$ to be given by

$$
S=\{k \in \mathbb{C}:[\operatorname{SP}(k)] \text { has a non-trivial solution }\} .
$$

The set of eigenvalues of $[\operatorname{SP}(k)]$ is $S^{d}$, where

$$
S^{d}=\left\{k \in \mathbb{C}: \exists \text { a non-trivial solution to }[\operatorname{SP}(k)] \text { with } \bar{\Phi}^{\prime} \rightarrow 0 \text { as } \bar{R} \rightarrow \infty\right\},
$$


and the continuous spectrum of $[\mathrm{SP}(k)]$ is $S^{c}$, where

$$
S^{c}=\left\{k \in \mathbb{C}: \exists \text { a non-trivial solution to }[\mathrm{SP}(k)] \text { with } \bar{\Phi}^{\prime} \nrightarrow 0 \text { as } \bar{R} \rightarrow \infty\right\},
$$

with the limits as $\bar{R} \rightarrow \infty$ considered as uniform for $-\alpha \leqslant \theta \leqslant 0$. Finally, we observe that

$$
S=S^{c} \cup S^{d}
$$

We now determine $\boldsymbol{S}$. Following Needham (2012), we obtain the following results.

THEOREM 1.

$$
S \subseteq\{k \in \mathbb{C}: \operatorname{Re}(k)>0\} \cup\{0\}=R_{+} .
$$

Proof. The proof requires that, for each $k \in \mathbb{C} \backslash R_{+}$, we establish that $[\mathrm{SP}(k)]$ has only the trivial solution. Let $k \in \mathbb{C} \backslash R_{+}$, and let $\bar{\Phi}^{\prime}: \overline{\mathcal{G}}_{\infty} \rightarrow \mathbb{C}$ be a solution to [SP $\left.(k)\right]$. Take any $R_{0}>0$ and set $\overline{\mathcal{G}}_{R_{0}}=\overline{\mathcal{G}}_{\infty} \backslash\left(R_{0}, \infty\right) \times[-\alpha, 0]$, and $\partial \mathcal{G}=\left\{(\bar{R}, \theta): 0 \leqslant \bar{R} \leqslant R_{0}, \theta=\right.$ $0\} \cup\left\{(\bar{R}, \theta): \bar{R}=R_{0},-\alpha<\theta<0\right\} \cup\left\{(\bar{R}, \theta): 0 \leqslant \bar{R} \leqslant R_{0}, \theta=-\alpha\right\}$, then Green's Theorem (see, for example, Kaplan (2002)), along with the regularity (3.59) and (A 1), gives

$$
\int_{\overline{\mathcal{G}}_{R_{0}}} \int\left\{\nabla \bar{\Phi}^{*} \cdot \nabla \bar{\Phi}^{\prime}\right\} \bar{R} \mathrm{~d} \bar{R} \mathrm{~d} \theta=\int_{\partial \mathcal{G}}\left\{\bar{\Phi}^{\prime *}\left(\nabla \bar{\Phi}^{\prime} \cdot \hat{\boldsymbol{n}}\right)\right\} \mathrm{d} S
$$

where superscript * denotes complex conjugation. It follows from (A 10) and (A 2) that

$$
\int_{\overline{\mathcal{G}}_{R_{0}}} \int\left(\left|\bar{\Phi}_{\bar{R}}^{\prime}\right|^{2}+\left|\frac{1}{\bar{R}} \bar{\Phi}_{\theta}^{\prime}\right|^{2}\right) \bar{R} \mathrm{~d} \bar{R} \mathrm{~d} \theta=\int_{-\alpha}^{0}\left(\bar{R} \bar{\Phi}^{\prime *} \bar{\Phi}_{\bar{R}}^{\prime}\right)_{\bar{R}=R_{0}} \mathrm{~d} \theta+\int_{0}^{R_{0}}\left(\bar{\Phi}^{\prime *} \frac{1}{\bar{R}} \bar{\Phi}_{\theta}^{\prime}\right)_{\theta=0} \mathrm{~d} \bar{R}
$$

which becomes, using (A 3),

$$
\int_{\overline{\mathcal{G}}_{R_{0}}} \int\left(\left|\bar{\Phi}_{\bar{R}}^{\prime}\right|^{2}+\left|\frac{1}{\bar{R}} \bar{\Phi}_{\theta}^{\prime}\right|^{2}\right) \bar{R} \mathrm{~d} \bar{R} \mathrm{~d} \theta-k \int_{0}^{R_{0}}\left(\left|\bar{\Phi}^{\prime}\right|^{2}\right)_{\theta=0} \mathrm{~d} \bar{R}=\int_{-\alpha}^{0}\left(\bar{R} \bar{\Phi}^{\prime *} \bar{\Phi}_{\bar{R}}^{\prime}\right)_{\bar{R}=R_{0}} \mathrm{~d} \theta
$$

for each $R_{0}>0$. We now introduce $u$ and $v$, where

$$
u(\bar{R}, \theta)=\operatorname{Re}\left[\bar{\Phi}^{\prime}(\bar{R}, \theta)\right], \quad v(\bar{R}, \theta)=\operatorname{Im}\left[\bar{\Phi}^{\prime}(\bar{R}, \theta)\right],
$$

after which we obtain from (A 12)

$$
\begin{aligned}
& \int_{\overline{\mathcal{G}}_{R_{0}}} \int\left(\left|\bar{\Phi}_{\bar{R}}^{\prime}\right|^{2}+\left|\frac{1}{\bar{R}} \bar{\Phi}_{\theta}^{\prime}\right|^{2}\right) \bar{R} \mathrm{~d} \bar{R} \mathrm{~d} \theta-k \int_{0}^{R_{0}}\left(\left|\bar{\Phi}^{\prime}\right|^{2}\right)_{\theta=0} \mathrm{~d} \bar{R} \\
& \quad=\frac{1}{2} R_{0} \frac{\mathrm{d}}{\mathrm{d} R_{0}}\left\{\int_{-\alpha}^{0}\left(\left|\bar{\Phi}^{\prime}\right|_{\bar{R}=R_{0}}^{2} \mathrm{~d} \theta\right)\right\}+\mathrm{i} R_{0} \int_{-\alpha}^{0}\left(u v_{\bar{R}}-u_{\bar{R}} v\right)_{\bar{R}=R_{0}} \mathrm{~d} \theta .
\end{aligned}
$$

Taking the real part of (A 14) gives

$$
\begin{aligned}
& \int_{\overline{\mathcal{G}}_{R_{0}}} \int\left(\left|\bar{\Phi}_{\bar{R}}^{\prime}\right|^{2}+\left|\frac{1}{\bar{R}} \bar{\Phi}_{\theta}^{\prime}\right|^{2}\right) \bar{R} \mathrm{~d} \bar{R} \mathrm{~d} \theta-\operatorname{Re}(k) \int_{0}^{R_{0}}\left(\left|\bar{\Phi}^{\prime}\right|^{2}\right)_{\theta=0} \mathrm{~d} \bar{R} \\
& =\frac{1}{2} R_{0} \frac{\mathrm{d}}{\mathrm{d} R_{0}}\left\{\int_{-\alpha}^{0}\left(\left|\bar{\Phi}^{\prime}\right|_{\bar{R}=R_{0}}^{2}\right) \mathrm{d} \theta\right\},
\end{aligned}
$$


for each $R_{0}>0$. Using (3.63) we can now write the right-hand side of (A 15), in the limit $R_{0} \rightarrow \infty$, as

$$
\begin{aligned}
& \frac{1}{2} R_{0} \frac{\mathrm{d}}{\mathrm{d} R_{0}}\left\{\int_{-\alpha}^{0}\left(\left|\bar{\Phi}^{\prime}\right|_{\bar{R}=R_{0}}^{2} \mathrm{~d} \theta\right)\right\} \\
& \quad=\frac{1}{2} R_{0} \frac{\mathrm{d}}{\mathrm{d} R_{0}}\left\{\int_{-\alpha}^{0}\left(\left|a_{k}\right|^{2} \frac{1}{R_{0}^{\pi / \alpha}} \cos ^{2} \frac{\pi}{2 \alpha}(\theta+\alpha)+O\left(\frac{1}{R_{0}^{\pi / \alpha+1}}\right)\right) \mathrm{d} \theta\right\} \\
& \quad=-\frac{\pi}{4}\left|a_{k}\right|^{2} \frac{1}{R_{0}^{\pi / \alpha}}+O\left(\frac{1}{R_{0}^{\pi / \alpha+1}}\right),
\end{aligned}
$$

which is bounded as $R_{0} \rightarrow \infty$. Since $\operatorname{Re}(k) \leqslant 0$, it then follows from (A 15) that

$$
\int_{\overline{\mathcal{G}}_{R_{0}}} \int\left(\left|\bar{\Phi}_{\bar{R}}^{\prime}\right|^{2}+\left|\frac{1}{\bar{R}} \bar{\Phi}_{\theta}^{\prime}\right|^{2}\right) \bar{R} \mathrm{~d} \bar{R} \mathrm{~d} \theta \leqslant \frac{1}{2} R_{0} \frac{\mathrm{d}}{\mathrm{d} R_{0}}\left\{\int_{-\alpha}^{0}\left(\left|\bar{\Phi}^{\prime}\right|_{\bar{R}=R_{0}}^{2} \mathrm{~d} \theta\right)\right\},
$$

and so the left-hand side of (A 17) is bounded and non-decreasing as $R_{0} \rightarrow \infty$, and thus has a finite non-negative limit as $R_{0} \rightarrow \infty$, so that

$$
\lim _{R_{0} \rightarrow \infty} \int_{\overline{\mathcal{G}}_{R_{0}}} \int\left(\left|\bar{\Phi}_{\bar{R}}^{\prime}\right|^{2}+\left|\frac{1}{\bar{R}} \bar{\Phi}_{\theta}^{\prime}\right|^{2}\right) \bar{R} \mathrm{~d} \bar{R} \mathrm{~d} \theta=\beta,
$$

for some $\beta \geqslant 0$. However, it now follows from (A 16) to (A 18) that $\beta \leqslant 0$, and so we conclude that $\beta=0$. We then have from (A 18) that

$$
\int_{\overline{\mathcal{G}}_{R_{0}}} \int\left(\left|\bar{\Phi}_{\bar{R}}^{\prime}\right|^{2}+\left|\frac{1}{\bar{R}} \bar{\Phi}_{\theta}^{\prime}\right|^{2}\right) \bar{R} \mathrm{~d} \bar{R} \mathrm{~d} \theta=0,
$$

for all $R_{0}>0$. The condition (A 19) (along with regularity (3.59)) then requires that $\bar{\Phi}^{\prime}(\bar{R}, \theta)=C$ for all $(\bar{R}, \theta) \in[0, \infty) \times[-\alpha, 0]$, for some constant $C \in \mathbb{C}$. However, since $k \neq 0$, we have from (A 3) that $C=0$, so that $\bar{\Phi}^{\prime}(\bar{R}, \theta)=0$ for all $(\bar{R}, \theta) \in[0, \infty) \times[-\alpha, 0]$, which is the trivial solution, and the proof is complete.

\section{THEOREM 2.}

$$
S \subseteq \mathbb{R}^{+} \cup\{0\} .
$$

Proof. Let $k \in R_{+} \backslash\left(\mathbb{R}^{+} \cup\{0\}\right)$, and $\bar{\Phi}^{\prime}: \overline{\mathcal{G}}_{\infty} \rightarrow \mathbb{C}$ be a solution to $[\operatorname{SP}(k)]$. We must show that $\bar{\Phi}^{\prime}$ is the trivial solution. We have from (3.63) that

$$
\bar{\Phi}^{\prime}(\bar{R}, \theta)=a \frac{1}{\bar{R}^{\pi / 2 \alpha}} \cos \frac{\pi}{2 \alpha}(\theta+\alpha)+O\left(\frac{1}{\bar{R}^{\pi / 2 \alpha+1}}\right),
$$

as $\bar{R} \rightarrow \infty$, uniformly for $-\alpha \leqslant \theta \leqslant 0$, with $a \in \mathbb{C}$ being a non-zero constant. We also have from (A 14), that

$$
\operatorname{Im}(k) \int_{0}^{R_{0}}\left(\left|\bar{\Phi}^{\prime}\right|^{2}\right)_{\theta=0} \mathrm{~d} \bar{R}+R_{0} \int_{-\alpha}^{0}\left(\overline{u v}_{\bar{R}}-\bar{u}_{\bar{R}} \bar{v}\right)_{\bar{R}=R_{0}} \mathrm{~d} \theta=0
$$


and

$$
\begin{aligned}
& \int_{\overline{\mathcal{G}}_{R_{0}}} \int\left(\left|\bar{\Phi}_{\bar{R}}^{\prime}\right|^{2}+\left|\frac{1}{\bar{R}} \bar{\Phi}_{\theta}^{\prime}\right|^{2}\right) \bar{R} \mathrm{~d} \bar{R} \mathrm{~d} \theta-\operatorname{Re}(k) \int_{0}^{R_{0}}\left(\left|\bar{\Phi}^{\prime}\right|^{2}\right)_{\theta=0} \mathrm{~d} \bar{R} \\
& =\frac{1}{2} R_{0} \frac{\mathrm{d}}{\mathrm{d} R_{0}}\left\{\int_{-\alpha}^{0}\left(\left|\bar{\Phi}^{\prime}\right|^{2}\right)_{\bar{R}=R_{0}} \mathrm{~d} \theta\right\} .
\end{aligned}
$$

Now, via (A 21), we have that

$$
\lim _{R_{0} \rightarrow \infty} R_{0} \int_{-\alpha}^{0}\left(\overline{u \bar{v}_{\bar{R}}}-\bar{u}_{\bar{R}} \bar{v}\right)_{\bar{R}=R_{0}} \mathrm{~d} \theta=0 .
$$

Since $\operatorname{Im}(k) \neq 0$ it then follows from (A 22) that

$$
\lim _{R_{0} \rightarrow \infty} \int_{0}^{R_{0}}\left(\left|\bar{\Phi}^{\prime}\right|^{2}\right)_{\theta=0} \mathrm{~d} \bar{R}=0
$$

and so

$$
\int_{0}^{R_{0}}\left(\left|\bar{\Phi}^{\prime}\right|^{2}\right)_{\theta=0} \mathrm{~d} \bar{R}=0
$$

for all $R_{0}>0$. Next, considering (A 23), (A 26), and (A 16), we have that

$$
\lim _{R_{0} \rightarrow \infty} \int_{\overline{\mathcal{G}}_{R_{0}}} \int\left(\left|\bar{\Phi}_{\bar{R}}^{\prime}\right|^{2}+\left|\frac{1}{\bar{R}} \bar{\Phi}_{\theta}^{\prime}\right|^{2}\right) \bar{R} \mathrm{~d} \bar{R} \mathrm{~d} \theta=0 .
$$

It then immediately follows that $\bar{\Phi}^{\prime}(\bar{R}, \theta)=0$, for all $(\bar{R}, \theta) \in[0, \infty) \times[-\alpha, 0]$, which is the trivial solution, and the proof is complete.

In fact $S=\mathbb{R}^{+} \cup\{0\}$. Clearly, $0 \in S$, since when $k=0, \bar{\Phi}^{\prime}(\bar{R}, \theta)=1$ for all $(\bar{R}, \theta) \in[0, \infty) \times[-\alpha, 0]$, solves $[S P(0)]$. Moreover John (1948) has established that $k \in S$ for all $k \in \mathbb{R}^{+}$, and in addition that, $S^{c}=\mathbb{R}^{+} \cup(0)$ whilst $\boldsymbol{S}^{d}=\varnothing$.

\section{REFERENCES}

Gallagher, M. T., Needham, D. J. \& Billingham, J. 2018 The initial development of a jet caused by fluid, body and free surface interaction with a uniformly accelerated advancing or retreating plate. Part 1. The principal flow. J. Fluid Mech. 841, 109-145.

John, F. 1948 Waves in the presence of an inclined barrier. Commun. Pure Appl. Maths 1 (2), 149-200.

Kaplan, W. 2002 Advanced Calculus, Addison-Wesley Higher Mathematics, vol. 5. Addison-Wesley.

KIng, A. C. \& Needham, D. J. 1994 The initial development of a jet caused by fluid, body and free-surface interaction. Part 1. A uniformly accelerating plate. J. Fluid Mech. 268, 89-101.

NeEdham, D. J. 2012 The initial development of a jet caused by fluid, body and free surface interaction. Part 4. The large-time structure. IMA J. Appl. Maths 77 (4), 451-472.

Needham, D. J., Billingham, J. \& King, A. C. 2007 The initial development of a jet caused by fluid, body and free-surface interaction. Part 2. An impulsively moved plate. J. Fluid Mech. 578, 67-84.

Needham, D. J., Chamberlain, P. G. \& Billingham, J. 2008 The initial development of a jet caused by fluid, body and free surface interaction. Part 3. An inclined accelerating plate. Q. J. Mech. Appl. Maths 61 (4), 581-614.

Sharp, D. H. 1984 An overview of Rayleigh-Taylor instability. Physica D 12 (13), 3-18.

VAN DyKe, M. 1964 Perturbation Methods in Fluid Mechanics, Applied Mathematics and Mechanics, vol. 8. Academic Press. 